Krypton and Xenon.-Moore ${ }^{1}$ isolated krypton and xenon in considerable quantities from the residues from 120 tons of liquid air. Calculated from the densities of the two gases the atomic weights are $\mathrm{Kr}=83.012$, and $\mathrm{Xe}=\mathrm{I} 30.7 \mathrm{O}$.

It will be seen from the evidence given above that few changes are needed in the table of atomic weights. Chromium, 52.0I, may be rounded off to 52, as compared with the 52.I formerly accepted. Arsenic becomes 74.96, in accordance with the work of Baxter and Coffin. The new values for krypton and xenon should also be adopted. As regards mercury, action may be deferred until more evidence is received.

$$
\begin{array}{ll}
\text { Signed, F. W. Clarke, } & \text { T. E. Thorpe, } \\
& \text { W. OstWai, }, \\
\text { G. Urbain. }
\end{array}
$$

[Contributton from the Chemical Laboratory of Harvard College.]

\title{
FURTHER INVESTIGATION CONCERNING THE ATOMIC WEIGHTS OF SILVER, LITHIUM AND CHLORINE.
}

BY THEODORE W, RICHARDS AND HOBART HURD WILIARD,

Received October 28, 1909 .

\section{PART I,-THE RATIO OF LITHIUM CHLORIDE TO SILVER CHLORIDE AND SILVER. \\ Introduction.}

The present uncertainty in the atomic weight of silver causes confusion and uncertainty throughout the whole table of atomic weights. Long ago Dumas ${ }^{2}$ pointed out that Stas, with all his care, had not succeeded in preparing pure silver, and accordingly that the atomic weights referred to silver need revision. Later, in reply to Dumas, Stas ${ }^{3}$ sought to determine the oxygen remaining in the metal and succeeded in finding only a trace. Hence for many years Dumas' criticism was unheeded. A careful study of Stas's experiments shows, however, that the metal which he used in these later trials had not been treated in the same way as that which he used in his work upon atomic weights and hence that the presence or absence of oxygen in the later samples proved little with regard to the earlier samples. As a matter of fact it was shown in 1903 in the chemical laboratory of Harvard University ${ }^{4}$ that Stas's silver

${ }^{1} \mathrm{~J}$. Chem. Soc, , 93, $218 \mathrm{x}$.

"Dumas, Ann. chim. phys., 14, 289 (1878).

${ }^{3}$ Stas, Oeuvres Completes, III, 106-I25 (Brusseis, I903).

"Richards and Wells, "A Revision of the Atomic Weights of Sodium and Chlorine," Carnegie Institution of Washington, Publication No. 28; ThIs Journal, 27, 459 (1905); Z, anorg. Chem., 47, 56 (1905). This paper is reprinted in full in "Experimentelle Untersuchungen über die Atomgewichte," by T. W. Richards, page 689 (Hamburg, 1909), and also in Chem. News, 93. 
must have been impure to the extent of at least 0.015 per cent. and that therefore his value for the atomic weight of this element, as well as of the others which had been referred to it, must have been in error. Clearly Stas's value for silver must be too large, but no attempt was made at that time to discover just how great the error might be, this proof being a somewhat complicated matter. Since that time increasing evidence has been presented, especially by Guye, tending to show that the discrepancy is serious. Because most other elements are referred to oxygen only through silver, on account of the insolubility of many silver salts and their consequent ease of precipitation, this uncertainty in the atomic weight of silver is one of very serious moment. Indeed a majority of the atomic weights cannot be referred to oxygen with precision until the relation between silver and oxygen is ascertained without doubt. ${ }^{1}$

To discover the most suitable method of attaining this end, and to obtain the greatest accuracy possible in the solution of the problem, was the object of the present research. We were fortunate in being able to profit by previous experience, many of the difficulties having already been surmounted in previous investigations carried out in this laboratory.

Before discussing the present investigation, it will be helpful to review briefly the work previously done relating to the oxygen-silver ratio. Unfortunately no compound of silver and oxygen exists of a nature suitable for exact work. The two common oxides of silver are far too unstable to yield results possessing any precision, and moreover, the amount of oxygen in even the higher of these oxides is a comparatively small percentage of the whole. Hence the necessary errors of the determination are greatly magnified in the calculation. For this reason it is necessary, as Stas realized, to study a compound of silver and oxygen which contains some third element, and various salts are available for the purpose. Stas himself used chiefly the chlorates, bromates, and iodates for this purpose, determining the ratio $3 \mathrm{O}: \mathrm{Ag}$ by multiplying together the values found by him for the following pairs of ratios, thus:

$$
\frac{3 \mathrm{O}}{\mathrm{KCl}} \cdot \frac{\mathrm{KCl}}{\mathrm{Ag}}=\frac{3 \mathrm{O}}{\mathrm{Ag}} ; \frac{3 \mathrm{O}}{\mathrm{AgCl}} \cdot \frac{\mathrm{AgCl}}{\mathrm{Ag}}=\frac{3 \mathrm{O}}{\mathrm{Ag}} ; \frac{3 \mathrm{O}}{\mathrm{AgBr}} \cdot \frac{\mathrm{AgBr}}{\mathrm{Ag}}=\frac{3 \mathrm{O}}{\mathrm{Ag}} ; \frac{3 \mathrm{O}}{\mathrm{AgI}} \cdot \frac{\mathrm{AgI}}{\mathrm{Ag}}=\frac{3 \mathrm{O}}{\mathrm{Ag}} \text {. }
$$

In each case the first ratio was obtained by the decomposition of a halate (i.e., a chlorate, bromate, or iodate) and the second ratio by the comparison of silver with a halide. If the exact identity of the halide obtained from each oxygen salt with that employed in the corresponding second process could be proved, and if the various substances had been pure, the method would have been an admirable one; but unfortunately

1 "Report International Committee on Atomic Weights, 1906," Guye and Ter Gazarian, Compt. rend., 143, 41 I (1906). Noyes, This Journal, 29, I719 (1907). 
neither of these important qualifications was completely attained. Moreover, other minor errors crept in to the work. ${ }^{1}$

The nitrate and sulphate of silver were also studied by Stas, and his figures concerning these substances may be used in a similar way. These researches have been recently repeated with greater precision in this laboratory, ${ }^{2}$ but here the outcome is complicated by the fact that the relation neither of nitrogen to silver nor of sulphur to silver is precisely known. Nitrogen, it is true, has been referred with much plausibility to oxygen, with the help of gas densities; but this is a physical rather than a chemical method of determining atomic weights. Excellent though it undoubtedly is as corroborative evidence, it cannot carry with it the conclusiveness which a chemical determination could furnish. On the other hand, the atomic weight of sulphur is far from certain.

Without attempting to give a complete historical discussion, the most important results of other investigations may be mentioned. The interesting and valuable results of Dixon and Edgar, ${ }^{3}$ of W. A. Noyes ${ }^{4}$ and $\mathrm{H}$. C. P. Weber and of Edgar alone ${ }^{5}$ upon the quantitative synthesis of hydrochloric acid furnish less direct evidence upon the matter in hand, for they need the introduction of two other ratios, namely, that of hydrogen to oxygen and that of chlorine to silver, in order to solve the problem. Thus:

$$
\frac{2 \mathrm{H}}{2 \mathrm{Cl}} \cdot \frac{2 \mathrm{C}}{2 \mathrm{Ag}} \cdot \frac{\mathrm{O}}{2 \mathrm{H}}=\frac{\mathrm{O}}{2 \mathrm{Ag}} .
$$

Even if the result were not burdened with all the errors which may reside in the additional figures, it would not be very satisfactory, because oxygen is compared with over thirteen times its weight of silver, and any error becomes greatly magnified in the calculation.

Simpler arithmetical manipulation is demanded by the recent interesting complete analysis by Guye and Fluss ${ }^{8}$ of nitrosyl chloride, $\mathrm{NOCl}$, but the result is still somewhat open to the last objection. Thus:

$$
\frac{\mathrm{O}}{\mathrm{Cl}} \cdot \frac{\mathrm{Cl}}{\mathrm{Ag}}=\frac{\mathrm{O}}{\mathrm{Ag}} \text {. }
$$

Here the weight of oxygen for a given weight of silver is double that in the last case.

A more promising method has been developed by Baxter, ${ }^{7}$ and carried

1 The best discussion of this subject is to be found in Brauner's admirable discussion on "The Fundamental Atomic Weights" in Abegg's "Handbuch der anorganischen Chemie," II, I, $177-182$.

"Publ. Carnegie Inst., Washington, 69.

Phil. Trans., 205, I69.

- This Journal, 30, I3 (1908).

- Proc. Roy. Soc., London (A) 81, 2 I6 (Igo8).

- Guye and Fluss, J. chim. phy's., 6, 732 (1908).

7Baxter and Tilley, Thrs Journal, 3I, 201 (I909). 
out with the assistance of $\mathrm{G}$. S. Tilley, namely, the direct analysis by decomposition of iodine pentoxide. In this way may be determined with considerable accuracy the ratio $\mathrm{I}_{2}: 5 \mathrm{O}$ and by multiplying the results by the ratio of silver to iodine, ${ }^{1}$ the ratio of $2 \mathrm{Ag}:{ }_{5} \mathrm{O}$ is at once obtained. Thus:

$$
\frac{5 \mathrm{O}}{2 \mathrm{I}} \cdot \frac{2 \mathrm{I}}{2 \mathrm{Ag}}=\frac{5 \mathrm{O}}{2 \mathrm{Ag}}
$$

If the second ratio were known with the greatest accuracy, this method would be an excellent one, but the peculiar nature of silver iodide has interfered with the attainment of quite as certain results concerning this substance as concerning the other silver halides.

The values obtained for silver from these various methods range from I07.92, based upon Edgar's value for chlorine and Morley's value for hydrogen, to I07.85, based upon Baxter and Tilley's work with iodine pentoxide, and it is safe to conclude that the truth lies between these extremes. Nevertheless, the range of uncertainty (0.07, or distinctly over one-twentieth of one per cent.) is far too great for a datum of such importance.

The necessity for further work upon the ratio of silver to oxygen is very evident, and the present paper recounts an extended investigation, protracted over two years, which has furnished entirely new results of a nature apparently more trustworthy than any that had gone before.

As a first step, all substances which might possibly be available for obtaining further knowledge of this very important ratio were scrutinized with the greatest care, in order to determine whether or not a better starting-point might be found. Evidently, from the foregoing remarks, the selection of the substance for this purpose depends upon more than one consideration. In the first place, it is important that the actual weights of the two elements to be compared should not be very different, so that the error in one may not be greatly magnified in a calculation of the other; hence, because the atomic weight of oxygen is much smaller than that of silver, the comparison must involve several atoms of oxygen with one of silver, if possible. For this reason the most highly oxygenated salts were studied first. It is, moreover, necessary to choose a salt of such a nature that the two ratios $\frac{\mathrm{MXO}_{y}}{\mathrm{MX}}$ and $\frac{\mathrm{MX}}{\mathrm{Ag}}$ may each be accurately determined. With respect to each requirement, the perchlorates seemed to furnish a peculiarly suitable starting-point. It is indeed remarkable that all previous investigators have chosen to work with chlorates in preference to the perchlorates, which are more stable, contain more oxygen, and are more easily obtained pure.

${ }^{1}$ Baxter, Proc. Am. Acad., 4I, 73 (I905). 
The choice among the perchlorates was determined by the other considerations which must always form an important factor in a choice of this kind, namely, the nature of the substances to be weighed. In order that accurate results may be obtained, it is obvious that all the substances whose precise weights come into consideration should be capable of complete purification and thorough drying, and the great difficulties attending these processes are governed largely by the specific nature of the substances. In order that a substance may be prepared in a pure state, it must be capable of easy crystallization in such a way as to free it from its usually attendant impurities; and in order that it may be dried, it must be capable of fusion without decomposition. There are no perbromates and the periodates are too unstable; among the perchlorates the only substance which corresponds to these specifications seems to be lithium perchlorate, which fuses at $236^{\circ}$ and loses no oxygen at $300^{\circ}$. Fortunately in other respects this substance is peculiarly well suited for the purpose. Its decomposition products, if any, can be accurately determined. It crystallizes well, and its solubility has a large temperature coefficient; therefore it may be easily prepared pure. It is not deliquescent or markedly hygroscopic in fairly dry air. It can be synthesized from the chloride merely by the evaporation of this salt with a slight excess of perchloric acid, the less volatile perchloric acid easily expelling the more volatile hydrochloric acid. Finally it contains over 60 per cent. of oxygen-probably more available oxygen than any other known compound, not excepting anhydrous hydrogen peroxide. Ten cubic centimeters of the salt, weighing about 24 grams, contain enough oxygen to occupy, under normal conditions, nearly ro liters, and all this oxygen is lost when the salt is converted into chloride. Lithium has the least atomic weight of any metal, and chlorine the least of any halogen which forms oxygen salts; therefore it is easy to see how this favorable circumstance comes to pass. Such a substance affords a far more satisfactory means of weighing a gas than any attempt to weigh it in an uncombined condition. Whın one considers the bulky apparatus necessary in weighing gases, with the attendant errors, and the unavoidable small weights of gas, it is easy to see the superiority of a process involving a solid salt, containing the gas in an exceedingly condensed form. The weighing of hydrogen absorbed by palladium, a method employed in the recent work on the synthesis of water, is an illustration of this point. In the present case, we may consider the lithium chloride as absorbing oxygen, but far more efficiently.

Lithium perchlorate was finally chosen, however, only after a careful study of the other perchlorates, in order to make certain that none of the others offered other advantages outweighing those peculiar to itself. Attention was first directed toward silver perchlorate, which is easily 
prepared by evaporating a solution of the nitrate with excess of perchloric acid, and expelling the free acid by heat. The salt is extremely deliquescent, and is difficult to dry without slight decomposition. Its fusing point is so high $\left(486^{\circ}\right)$ that it cannot be completely fused before violent decomposition begins, and for the same reason an accurate determination of the water remaining in the salt dried at a lower temperature, would be difficult, if not impossible. Silver perchlorate was, therefore, abandoned, although it has one great advantage-it is easily converted into chloride in a current of dry hydrochloric acid gas even at ordinary temperatures, the process involving no transfer of material. The reaction is not a reduction, anhydrous perchloric acid being formed.

The alkaline perchlorates were next investigated. Potassium perchlorate cannot be fused without rapid decomposition. Sodium perchlorate has a lower fusing point $\left(482^{\circ}\right)$, but it decomposes slowly at this temperature, and hence cannot be accurately weighed. All indications therefore pointed to lithium perchlorate, whose virtues have been already described.

The ratio of silver to oxygen cannot be determined from this salt, however, until that of lithium chloride to silver is known. Thus, writing the relation in a manner analogous to that employed in the case of the chlorates:

$$
\frac{4 \mathrm{O}}{\mathrm{LiCl}} \cdot \frac{\mathrm{LiCl}}{\mathrm{Ag}}=\frac{4 \mathrm{O}}{\mathrm{Ag}} \text {. }
$$

Our knowledge of this second ratio ( $\mathrm{LiCl}: \mathrm{Ag}$ ) depended upon the doubtful work of Stas, and this was at best freighted with the known errors entering into this work upon potassium and sodium chlorides. Hence, an essential step in the new work was the determination of this ratio, and incidentally of the true atomic weight of lithium. Even if Stas's value for lithium had been precise, however, instead of as we found it, nearly a whole per cent. in error, there would still have been good reason for making this determination anew with the same material used in our work with the perchlorate ratio. If the same sample of lithium chloride is used in determining both ratios, it is evident that the value obtained for the oxygen-silver ratio is independent of any other atomic weight, and that the weight of the alkaline chloride is eliminated. It matters not whether this chloride is free from other alkaline metals or contains them. To use an impure lithium salt would be, of course, a waste of time; from such a substance the atomic weight of lithium could not simultaneously be found.

The methods of determining these two ratios $\frac{\mathrm{LiCl}}{\mathrm{Ag}}$ and $\frac{4 \mathrm{O}}{\mathrm{LiCl}}$ are so widely different that they will be treated separately. While the ratio of silver to a chloride has so frequently been carried out in this laboratory that the operation presents no difficulties, the method for determining 
the amount of oxygen in the perchlorate was solved only after a long and careful research. The former of the two processes naturally precedes the latter; accordingly the determination of the equivalent of lithium chloride, referred both to silver and to silver chloride will be found in the first section of this paper. On the other hand the second section of the paper deals with the preparation of the perchlorate and the determination of the oxygen which it contains.

We take pleasure in expressing our obligation to the Carnegie Institution of Washington for generous pecuniary assistance throughout the course of the work.

\section{History of the Revisions of the Atomic Weight of Lithium.}

A glance at the following table, which shows the results so far obtained, reveals wide discrepancies. ${ }^{1}$

\begin{tabular}{|c|c|c|c|c|c|}
\hline & & & & Atomic & weight. \\
\hline & Name. & Year. & & $\mathrm{Ag}=\mathrm{r0} 7.93$. & $\mathrm{Ag}=107.88$ \\
\hline I. & Atfvedson & 1817 & $\mathrm{LiCl}: \mathrm{AgCl} \ldots \ldots \ldots \ldots$ & . IO. I & $\ldots$ \\
\hline 2. & Vauquelin & 1818 & $\mathrm{Li}_{2} \mathrm{SO}_{4}: \mathrm{BaSO}_{4} \ldots \ldots \ldots$ & .. 9.3 & $\ldots$ \\
\hline 3. & Gmelin & 1819 & Analysis of $\mathrm{Li}_{2} \mathrm{CO}_{3} \ldots \ldots$ & $\ldots 9.6$ & $\ldots$ \\
\hline & & & Analysis of $\mathrm{Li}_{2} \mathrm{SO}_{4} \ldots \ldots$ & $\ldots 10.7$ & $\ldots$ \\
\hline & & & Gmelin assumes......... & $\cdots \quad 7.65$ & $\ldots$ \\
\hline 4. & Kralovansky & 1827 & $\mathrm{Li}_{2} \mathrm{SO}_{4}: \mathrm{BaSO}_{4} \ldots \ldots \ldots$ & $\ldots 10.1$ & $\cdots$ \\
\hline 5 . & Hermann & 1829 & $\mathrm{Ii}_{2} \mathrm{CO}_{3}: \mathrm{CO}_{2} \ldots \ldots \ldots$ & $\ldots 6 . I$ & $\ldots$ \\
\hline & & & $\mathrm{Ii}_{2} \mathrm{SO}_{4} \cdot \mathrm{BaSO}_{4} \ldots \ldots \ldots$ & 6.1 & $\cdots$ \\
\hline 6. & Berzelius & 1829 & $\mathrm{Li}_{2} \mathrm{SO}_{4}: \mathrm{BaSO}_{4} \ldots \ldots \ldots$ & 6.69 & $\ldots$ \\
\hline 7. & Hagen & 1839 & $\mathrm{Li}_{2} \mathrm{SO}_{4}: \mathrm{BaSO}_{4} \ldots \ldots \ldots$ & 6.6 & $\ldots$ \\
\hline 8. & Mallet & 1856 & $\mathrm{IiCl}: \mathrm{AgC1} \ldots \ldots \ldots$ & 6.96 & $6.96^{*}$ \\
\hline & & & $\mathrm{I}, \mathrm{iCl}: \mathrm{Ag} \ldots \ldots \ldots \ldots$ & 6.92 & $6.92 *$ \\
\hline 9. & Troost & 1857 & $\mathrm{Li}_{2} \mathrm{CO}_{3}$ preliminary value. & 6.5 & $\ldots$ \\
\hline 10. & Mallet & 1859 & $\mathrm{Li}_{6} \mathrm{SO}_{4}: \mathrm{BaCl}_{2} \ldots \ldots \ldots$ & 7.01 & $\cdots$ \\
\hline II. & Dieh1 & 1862 & $\mathrm{Li}_{2} \mathrm{SO}_{4}: \mathrm{BaSO}_{4} \ldots \ldots \ldots$ & 7.04 & $\cdots$ \\
\hline & & & $\mathrm{Li}_{2} \mathrm{CO}_{3}: \mathrm{CO}_{2}, \ldots \ldots \ldots$ & 7.03 & $7.03^{*}$ \\
\hline I2. & Troost & 1862 & $\mathrm{Li}_{2} \mathrm{CO}_{3}: \mathrm{CO}_{2} \ldots \ldots \ldots$ & 7.00 & $7.00^{*}$ \\
\hline & & & $\mathrm{LiCl}: \mathrm{AgCl} \ldots \ldots \ldots \ldots$ & 6.96 & 6.96 \\
\hline & & & $\mathrm{I}_{4} \mathrm{i}_{2} \mathrm{CO}_{3}: \mathrm{I}_{2} \mathrm{i}_{2} \mathrm{SO}_{4}$ (one ex & ex- & \\
\hline & & & F & 7.13 & $\ldots$ \\
\hline 13. & Stas & I 865 & $\mathrm{LiCl}: \mathrm{Ag} \ldots \ldots \ldots \ldots$ & 7.006 & $7.003^{*}$ \\
\hline & & & Stas himself calculated... & 7.022 & $\ldots$ \\
\hline & & & $\mathrm{LiCl}: \mathrm{LiNO}_{3} \ldots \ldots \ldots$ & 6.92 & 6.96 \\
\hline I 4 . & Dittmar & 1889 & $\mathrm{Ii}_{2} \mathrm{CO}_{3}: \mathrm{CO}_{2} \ldots \ldots \ldots$ & 6.89 & 6.89 \\
\hline
\end{tabular}

'This table is taken from Brauner's admirable chapter on this subject in Abegg's "Handbuch der anorg. Chemie," II, $\mathbf{r}, 106$. The references to the original papers are as follows: I. Schweigg. Journ., 22, 93 (1817). 2. Ann. chim. phys., 7, 284 (1818). 3. Gilb. Ann., 72, 399 (1822). 4. Schweigg. Journ., 54, 231 (1827). 5. Pogg. Ann., 15, 480 (1829). 6. Ibid., 17, 379 (1829). 7. Ibid., 48, 361 (1839). 8. Sill, Am.J.Sci. [2], 22, 349 (1856). 9. Ann. chim. phys. [3], 51, III (1857). Io. Sill, Am. J. Sci. [2], 28, 349 (I859). II. Lieb. Ann., I2I, 93 (1862). I2. Compt. rend., 54, 366 (1862). I3. Stas, Oeuvres Completes, 1, 7 Io and 717 . 14. Proc. Roy. Soc. Edinburgh, 35. II, 429 . 
All excepting one of these determinations was made more than forty years ago, and the most recent, made twenty years ago, involved the use of a questionable method. Hence it is clear that a redetermination of the atomic weight would be desirable, even if it were not essential for the precise evaluation of silver and oxygen with the help of lithium perchlorate. Lithium alone among the five alkali metals has not received reasonable attention in this respect in recent years.

Of these twenty-three values found for the atomic weight of lithium, only those given in the last column of the table referred to the standard $\mathrm{Ag}=107.88$ are worthy of serious consideration. A few words discussing their merits and defects, in chronological sequence, will not be misplaced.

Mallet in 1856 purified his lithium chloride by dissolving it in alcohol and ether, but Stas has shown ${ }^{1}$ that by this method the salt cannot be separated from the chlorides of the other alkaline metals. He fused and weighed it in a covered platinum crucible-a process which must have given an alkaline product, in spite of the fact that ammonium chloride was added. Moreover, the hygroscopic nature of the chloride prevents it from being exactly weighed without greater protection from laboratory moisture. In two experiments the silver chloride was weighed, and in one the solution was titrated against a known weight of silver, following the method of Pelouze. The latter method in its old form gives too low results. The various errors seem to have counterbalanced one another, for the average value, 6.94, is almost exactly correct. This happy result could hardly have been foreseen, however.

Diehl purified his lithium by repeated precipitation with ammonium carbonate, thirty precipitations being required to free it from sodium. The lithium carbonate was then dissolved in sulphuric acid and treated with barium hydroxide, the lithium hydroxide thus obtained being converted into carbonate. This was dried at $130^{\circ}$ and decomposed in a Bunsen apparatus by means of dilute sulphuric acid, the carbon dioxide being determined by the loss in weight of the apparatus. Four experiments were performed, the average value obtained being 7.03 , but no vacuum corrections were applied, nor was a counterpoise used in weighing. The possibilities of uncertainty are so great that no one to-day would be disposed to accept the result without question.

Troost purified lithium carbonate by repeatedly dissolving it in cold water saturated with carbon dioxide and reprecipitating it by boiling the solution. In two experiments it was dried at $100^{\circ}$, mixed with powdered, ignited quartz, and the carbon dioxide determined by the loss of weight on heating. In one experiment it was converted into the sulphate. In two experiments lithium chloride was dehydrated in dry hydrochloric acid gas, which was afterwards displaced by dry air. The chlorine was

'Stas, Oeuvres Completes, r, 691. 
determined by weighing as silver chloride, and the mean value obtained for the atomic weight was 6.96. This outcome possesses the virtues and defects of Mallet's work.

Dittmar purified lithium carbonate by the method of Troost, but did not obtain a pure salt. He fused it in a current of dry carbon dioxide, the pressure varying from one to two atmospheres. By this means the formation of oxide was prevented. The carbonate was decomposed by dilute sulphuric acid, the gas evolved being absorbed by potassium hydroxide and weighed. The work was ingenious but very crude; the mean of ten results varying more than one-half per cent. was 6.89 .

Stas's work was far more careful than any cited above, although the final result was really not as accurate. He purified his lithium salt according to the methods of Diehl and of Troost. After removing most of the other metals by dissolving the chloride in alcohol and ether, the lithium was precipitated with ammonium carbonate seven times before it was found to be free from sodium. The lithium in the mother liquors was precipitated as carbonate, and this was purified by conversion into soluble bicarbonate, and reprecipitation of the solution by boiling - a very troublesome operation requiring several days. Two such treatments gave a product free from sodium. Both methods are extremely wasteful, I200 grams of fairly pure carbonate yielding only 145 grams of the purest salt. This was converted into chloride by heating in a platinum boat to $175^{\circ}$ in a current of dry hydrochloric acid gas. The chloride was then maintained in a state of fusion for twenty minutes, and finally cooled in nitrogen, after which it was weighed in a vacuum. The solid salt was subsequently dropped into a solution containing the theoretical amount of silver dissolved in nitric acid, and the titration was carried on exactly as in the work on sodium chloride. The errors incident to this process have been pointed out by Richards and Wells. ${ }^{1}$ Impurity in the silver and occlusion of silver nitrate by silver chloride tended to lower the atomic weight; impurity in the lithium chloride, and the error of method of determining the end point, tended to raise it. Stas himself calculated as the mean atomic weight of lithium from three experiments 7.022 , but this becomes 7.003 if modern values for silver and chlorine are substituted.

$\mathrm{He}$ also determined the ratio $\mathrm{LiNO}_{3}: \mathrm{LiCl}$ by dissolving the chloride in water in a large flask, evaporating with excess of nitric acid, and finally fusing the nitrate. Using modern values for nitrogen and chlorine, the atomic weight of lithium calculated from the mean of three experiments, is $6.96 \mathrm{I}$-a value much more nearly correct than the other.

Stas always found his lithium chloride alkaline to litmus, even when it had been heated in hydrochloric acid until half the salt had volatilized.

${ }^{1}$ This Jolrnal, 27, 459 (1905). 
It is difficult to account for this fact, yet it helps to explain the excessively high value for the atomic weight which he obtained. His lithium chloride volatilized in hydrochloric acid without leaving any residue, but this would not preclude the possibility that chlorides of the other alkalimetals were present. In some later work on lithium, ${ }^{1}$ he failed to obtain, by the means previously employed, a sample of lithium carbonate which would not show the sodium line upon very careful spectroscopic examination. It was necessary to volatilize in the oxyhydrogen flame, one-third or one-half of the carbonate, in order to obtain a residue of oxide absolutely free from sodium. From this oxide, he was unable to prepare pure lithium chloride, and he remarked that he believed it impossible to obtain that salt absolutely free from sodium unless every trace of dust could be excluded. It is obvious that the purification of lithium salts demands further attention.

Up to the present time, the most accurate work on this element has been usually supposed to be that of Stas. Since the errors in his work on the chlorides of potassium, sodium, and lithium are similar, and his values for the first two metals are too high, we should naturally expect the same to be true of lithium. The error was quite noticeable in the case of potassium, still more pronounced with sodium, and, as will be shown, was greatest of all with lithium.

In reviewing the foregoing results, which vary so widely, it seemed clear that the reason for the incompatibility of the values obtained for lithium, was to be sought not only in the methods of analysis, but also in the impurity of materials. Accordingly the first step of the present research was a careful study of the various practicable methods of purifying salts of lithium. The next procedure was obviously the application to the purest lithium material of the methods already so thoroughly studied in this laboratory in the cases of sodium and potassium. Some of the details of these methods were improved, and these improvements will be described in due course.

\section{Balance and Weighing.}

The excellent and sensitive Troemner balance which has served in many similar researches was used in the present work. Successive weighings of the same object rarely differed more than $0.02 \mathrm{mg}$.

The Sartorius gold-plated brass weights and rider were standardized from time to time by the method usually used at Harvard. ${ }^{2}$ All weighings were made by substitution, using a counterpoise similar to the object which was being weighed, so that the weights required were never large in amount, and the influence of changes in atmospheric conditions was negligible.

1 Oeuvres Completes, III, 324.

${ }^{2}$ Richards, This Journal, 22, I44 (I900). 
The vacuum corrections applied were as follows, the density of the weights being 8.3 .

\begin{tabular}{|c|c|c|}
\hline & Density. & Vac. cor. per gram. \\
\hline Silver......... & I0. 49 & -0.000030 \\
\hline Silver cbloride.... & $5 \cdot 56$ & +0.0000732 \\
\hline Lithium chloride" & 2.068 & +0.000436 \\
\hline Lithium perchlorat & 2.428 & +0.000350 \\
\hline
\end{tabular}

\section{The Preparation of Materials.}

All the solid, liquid and gaseous materials used in this research were purified with the greatest care. The most insidious sources of impurity in work of this kind are dust and the various gases sometimes contained in the air of the laboratory, and the most efficient methods of purification may fail to give a pure product unless careful attention is paid to this fact. The presence of dust, which always contains sodium, was especially noticeable in the preparation of pure lithium salts, where sodium was the element most difficult to remove. It was found that a lithium salt free from sodium, after being recrystallized in the usual way, always acquired traces of this element. The air of the room was, therefore, kept as pure as possible, and all evaporation, and handling of solutions and salts in the final work, were conducted in a large glass case. Vessels containing pure material were always kept covered and under bell jars when not in use. When it was necessary to work under the hood, a large, clean glass plate was suspended above the apparatus, to protect it from particles of dust falling from above. All heating was conducted electrically in order to avoid the deleterious effects of products of combustion.

Since most of the heating apparatus was constructed in this laboratory during the progress of the work, a brief description of it may not be inappropriate. The resistance material was "nichrome," an alloy of nickel, iron and chromium, which withstands fairly well a temperature of $1000^{\circ}$. It was generally used in the form of a ribbon, 1.5 to 3 millimeters wide. A small electrical stove or hot plate for conducting evaporations was made by winding this ribbon on a quartz plate, which was then covered with another thin plate of the same material, and the whole suitably mounted and insulated with asbestos. Another form, particularly adapted for heating flasks and dishes, was made by winding layers of wet sheet asbestos and the ribbon concentrically till the desired size was obtained; the roll, while still moist, was then pressed against the vessel to be heated and shaped to fit it. When dry, the mass of asbestos with its embedded ribbon, retained its form, and was mounted on asbestos board. A drying oven was made by winding a three-liter beaker with the ribbon, and jacketing it with heavy asbestos. By cutting through this jacket at one place, a window was formed, through which the interior could be observed.

${ }^{1}$ Baxter, Am. Chem. J., 3r, 558 (I9O4). 
A temperature of $400^{\circ}$ was easily obtained. A porcelain tube for fusing silver was wound with the ribbon and then covered with thick asbestos. When it was desired to heat a glass tube, and at the same time observe the interior, the ribbon was left bare, and the convolutions were far enough apart so as not appreciably to obstruct the view. The value of electrical heating, both as to cleanliness and wide range of application, was emphasized in the present research.

The vessels used in purifying materials were usually of quartz or platinum. Where glass was unavoidable, the best Jena glass was employed.

Water.-Distilled water of the laboratory was twice redistilled, once from alkaline permanganate and once alone, using pure tin condensers, without rubber or cork connections. ${ }^{1}$ "In special cases the water was condensed and collected wholly in platinum. Dust was carefully excluded by passing the end of the condenser through a hole blown in the bulb of a small flask, which rested on the mouth of the large Jena flask in which the water was collected and stored. Water was always distilled just before use.

Nitric Acid.-Carefully tested pure nitric acid of commerce was redistilled, using a platinum condenser. When required to be free from chlorine the first portion was rejected and the acid redistilled until it showed no trace of this element when examined in the nephelometer.

Hydrochloric Acid.-The pure acid of commerce was boiled with a little pure potassium permanganate, and then repeatedly distilled, using a quartz condenser.

Ammonium Fluoride--Baker's best hydrofluoric acid, which was already very pure, was distilled once in a platinum retort, and neutralized by distilling into it, through a platinum tube, pure ammonia from a concentrated solution of the gas.

Ammonium Carbonate.-The purest commercial salt was dissolved in water containing ammonia, and distilled in a platinum retort. Any trace of impurities contained in the product must have been volatile ones, which could exercise no harmful effect in the present case.

Hydrazine Hydrate.-This substance was prepared by distilling to dryness in platinum a mixture of hydrazine sulphate and potassium hydroxide in solution, using double the theoretical quantity of the latter substance. The resulting solution was distilled again from the small residue of alkali which had been carried over into it; the dilute portion coming over first was distilled yet once more in order to concentrate it, the first fractions, containing almost nothing but water being rejected. The substance was collected and preserved wholly in platinum, as were also the ammonia and ammonium fluoride.

Perchloric Acid.-This was prepared by a modification of the method

I Richards, Proc. Amer. Acad , 30, 380 (1894); Chem.-Ztg., 1907, No. 73, p. 899. 
of Kreider. ${ }^{1}$ Since sodium perchlorate can now be obtained in large quantities, the preparation of the stable hydrated acid is a comparatively simple operation, quite free from the dangers and difficulties involved in preparing the unstable anhydrous compound.

A saturated solution of sodium perchlorate was poured into two and one-half times its volume of pure concentrated hydrochloric acid (density, I.20). After thorough agitation the sodium chloride was allowed to settle, the clear liquid was siphoned off, and the sodium chloride drained in a funnel by suction, and washed with a little more acid. The solution, containing hydrochloric and perchloric acids, with a little salt of sodium, was evaporated until dense white fumes of perchloric acid began to appear. The liquid was then found to be entirely free from hydrochloric acid. At this point the concentration of the perchloric acid is approximately that of the dihydrate-about 70 per cent. If distilled under ordinary pressure, where it boils at $203^{\circ}$, there is considerable decomposition, involving a loss of perhaps ro per cent. The crude acid was therefore usually distilled under $I_{5}$ to $200 \mathrm{~mm}$. pressure, with almost no decomposition, but for the final distillation a pressure of $I_{5}$ to $20 \mathrm{~mm}$. was not exceeded. At first the acid was condensed in a platinum condenser bent at right angles and having its upper end slightly conical to fit into the constricted neck of a two-liter flask of resistant glass. The constriction was carefully ground until the platinum fitted it tightly. It was found that this joint held a vacuum very effectively after the flask and condenser had been pressed firmly together. The other end of the condenser passed through a rubber stopper fitted into a hole in a bell jar containing the platinum dish to receive the distillate. While the excess of water was being distilled off, the acid bumped rather badly; but when the concentration of the acid became that of the constant boiling mixture, this distilled rapidly and smoothly without any sign of ebullition. The yield is practically the theoretical.

Even when distilled under a pressure of $15 \mathrm{~mm}$. the acid had a faint odor, resembling that of the oxides of chlorine. It seemed possible, therefore, that the hot liquid might attack slightly the platinum condenser, although no evidence of such an effect was observed. Accordingly, in preparing the purest acid, we decided to condense and collect it in quartz vessels. To the quartz condenser was attached the half-liter distilling flask, into the neck of which was fused a dropping funnel, terminating in a capillary at the bottom of the flask. In this way acid could be introduced continuously during the distillation. The connection between the flask and the quartz condenser gave much trouble. At first a carefully ground joint was used, the quartz forming the inner portion,

${ }^{1}$ Z. anorg. Chem., 9, 343 (1895); Am. J. Sci. [3], 49, 443. Kreider and Breckenbridge, Z. anorg. Chem., I3, I6I (1896); Am. J. Sci. [4], 2, 263. 
but it was found that since the coefficient of expansion of glass is far greater than that of quartz, such a joint will invariably crack, even when exposed to a change of temperature of only $75^{\circ}$. If the outer portion is glass, it cracks on cooling; if quartz, on heating. The only possible joint between these two materials which will allow expansion and contraction, is one having two flat faces ground together as in Fig. I.

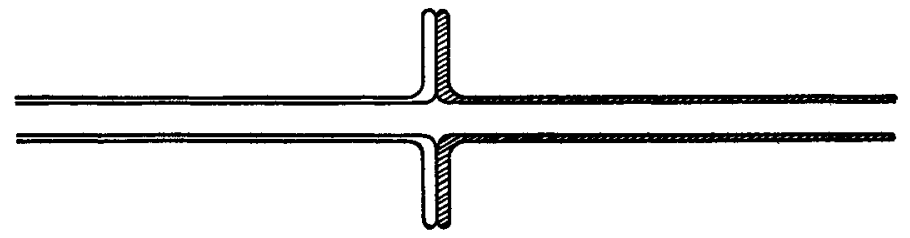

Fig. I.

Such a joint is easily made by forming a collar on the end of each tube, grinding them until perfectly plane, and finally polishing with oil and rouge. The two faces are held tightly together by a rubber band or a spring. Such a joint, without any lubricant, held a vacuum very effectively, and was entirely unaffected by changes of temperature. The joint was so placed that any condensed liquid coming into contact with the glass part would run back into the distilling flask.

After at least three previous distillations, the acid was finally distilled through the quartz condenser directly into a quartz flask, and all possibility of introducing impurity in this process was avoided.

The question of the purity of the acid thus obtained was carefully investigated. All the possible impurities must be those present in the sodium perchlorate and hydrochloric acid, and the only acids likely to remain after concentrating the crude acid, are sulphuric and phosphoric; the others are either destroyed or volatilized.

In order to determine how readily sulphuric acid could be separated from perchloric, a mixture of the latter with two per cent. of sulphuric acid was fractionated. After three distillations in which only to per cent. was rejected, 90 per cent. of the last distillate proved to be entirely free from sulphuric acid and 9 per cent. more contained so little that the residue left after evaporating off all but I $\mathrm{cc}$, showed a barely visible opalescence upon the addition of barium chloride. Phosphoric acid is even easier to remove. The residue remaining after the distillation of the crude acid from 8000 grams of sodium perchlorate, consisting mainly of sodium salts, contained a little sulphate and a trace of phosphate, but the last $20 \mathrm{cc}$. of acid distilled from this showed no trace of phosphoric acid.

Since the acid was never distilled less than four times, each time rejecting a small residue, its purity was evidently quite beyond reproach. If 
further proof was needed, this was furnished by the fact that the residue of $5 \mathrm{cc}$, remaining after distilling off about 8500 grams of acid (previously twice distilled) and which must have contained practically all the sulphuric acid present in the entire amount, showed only the merest trace of it, not over 0.0001 gram.

Silver.--The preparation of pure silver has been fully described by Richards and Wells ${ }^{1}$ and the details need not be repeated here. Four samples were prepared.

Sample A. Pure silver nitrate was recrystallized five times from dilute nitric acid, using vessels of Jena glass. The crystals were centrifugally drained in platinum funnels. The silver was then precipitated by ammonium formate, prepared from redistilled ammonia and formic acid, and after thorough washing, it was fused on pure lime in a current of dry hydrogen, generated by the action of "hydrone" on water. The boat was provided with partitions, so that buttons of a suitable size were formed. These were etched with dilute nitric acid, washed, and dried in a vacuum at $400^{\circ}$.

Sample B was prepared in a similar way except that it was recrystallized twice in glass and four times in platinum, and the reduction with formate was conducted in a platinum dish.

Sample $C$ was obtained by precipitating the mother liquors from A twice successively with formate, and then fusing on pure lime with a blast lamp.

Sample D was prepared from pure silver nitrate which had been once recrystallized, precipitated with formate, and fused on lime in hydrogen. This and sample $\mathrm{C}$ were used only as the source of silver nitrate in the precipitation of silver chloride to be weighed.

Experiments were made to determine whether the platinum dish was attacked by the hot nitric acid used to dissolve the silver nitrate, the dish being weighed before and after the operation. There was no loss in weight when the contents of the dish were stirred with a smooth glass rod, but when a platinum spatula was used, and the stirring was very vigorous, a loss of $0.24 \mathrm{mg}$. was observed, doubtless due to abrasion by its rather sharp edges. The precipitation of silver by formate caused no change in the weight of the dish.

Lithium Chloride.-The most common impurities in lithium salts and likewise the most difficult to eliminate are those elements most nearly related to it-the metals of the alkalies and alkaline earths. In the material which formed the source of the lithium chloride used in this work, the only impurities present in appreciable amounts were iron, calcium, potassium, sodium and probably magnesium. It is true that

1 This Journal, 27, 472 (1905).

2 This is the trade name for an alloy of sodium and lead. 
none of the latter was ever found, but this is probably due more to the difficulty of detecting small traces of it in the presence of large quantities of lithium, than to its entire absence. In Kahlbaum's lithium chloride, which formed part of the original material, no calcium, potassium or magnesium could be found. Since the iron was very easily removed, the impurities whose separation demanded careful investigation were potassium, sodium, calcium and magnesium. The relative effects of these on the atomic weight of lithium vary in the order given, that of potassium being the greatest.

Attention was first turned to the methods of eliminating potassium and sodium, which go together. Those employed by previous investigators were either inadequate or extremely wasteful. To the first class belongs that of dissolving the chloride or nitrate in a mixture of alcohol and ether. This was found to be effective in removing the greater part of the sodium from a crude chloride, especially if the solution were treated with hydrochloric acid gas until part of the lithium chloride was precipitated; but aside from this, it has no value. Precipitation by means of ammonium carbonate is effective, but the yield is very small, since only half of the lithium can be precipitated in one operation, owing to the solvent action of the ammonium salts. The conversion of the normal carbonate into bicarbonate demands too great a volume of solution, and is entirely too tedious where large quantities are required.

The most common method of purification - the recrystallization of soluble salts-seems to have received no attention, and accordingly various salts were tested with regard to their fitness for this purpose. With lithium chloride, the amount of sodium steadily decreased upon successive recrystallization until only a trace remained, but this trace was very hard to remove. Moreover, the solubility of the salt is so great that the process is very wasteful. The oxalate was almost useless in this respect. Recrystallization of the formate was fairly effective, but its electrolytic oxidation to carbonate was more so. Both the perchlorate and nitrate gave good yields, and the elimination of sodium was rapid, especially in the case of the nitrate. Care must be taken, however, to crystallize this latter salt below $29.6^{\circ}$, the transition point between the hydrated salts $\mathrm{LiNO}_{3} .1 / 2 \mathrm{H}_{2} \mathrm{O}$ and $\mathrm{LiNO}_{3} \cdot 3 \mathrm{H}_{2} \mathrm{O}$. Above $6 \mathrm{I}^{\circ}$ the anhydrous salt crystallizes, and under these conditions the separation from sodium is poor. The perchlorate crystallizes as $\mathrm{LiClO}_{4} \cdot 3 \mathrm{H}_{2} \mathrm{O}$.

These two salts were, therefore, selected as giving the most favorable results, but even with their help it is almost impossible to eliminate the last traces of sodium. It was evident that some method involving precipitation would be necessary. A search among the insoluble salts of lithium showed that the solubility of the fluoride is only 2.7 grams per liter-one-fifth that of the carbonate-and that this solubility is not ap- 
preciably affected by the presence of ammonium salts. Sodium fluoride is sixteen times, and potassium fluoride three hundred and forty times more soluble. Evidently the precipitation of lithium fluoride by ammonium fluoride ought therefore to be an extremely efficient means of removing sodium, without appreciable loss of lithium, and this was found to be true. Solutions of ammonium fluoride and of a lithium salt were run slowly into boiling water in a platinum dish, with constant stirring, taking care to avoid any great excess of either. The lithium fluoride formed under these conditions was coarsely crystalline and the precipitation was practically complete. The precipitate was transferred to platinum funnels and washed several times with water, using centrifugal drainage. Such washing has been shown to be very effective. ${ }^{1}$ If the original material contained comparatively little sodium, this precipitate was found to be entirely free from it, upon careful spectroscopic examination, and a repetition of the precipitation was sufficient to attain this end, even with very crude material. The pure fluoride was then converted into either nitrate or perchlorate by heating in a platinum retort with the corresponding acid--the distillate when perchloric acid was used being pure hydrofluoric acid, which was used again. When nitric acid was used, three or four times the theoretical quantity was required, owing to the slight difference in the boiling points of the acids. Thus it was possible to obtain, rapidly and without appreciable loss, lithium nitrate or perchlorate absolutely free from sodium. Some difficulty was experienced when making the spectroscopic tests, in obtaining a flame free from sodium, but if the air of the room was not dusty, this could be accomplished by using a small blast flame, supplied with pure washed air.

This method does not, however, eliminate calcium and magnesium, since the fluorides of both these metals are less soluble than that of lithium. Previous treatment of the solution with lithium fluoride is not very effective, but the addition of ammonium oxalate removes nearly all the calcium. The addition of lithium carbonate is yet better, only traces of calcium and magnesium remaining. These may be wholly separated by the recrystallization of the nitrate or the perchlorate. From a sample of lithium nitrate to which had been added so per cent. each of lithium sulphate, and calcium and magnesium as nitrates, there was obtained after four recrystallizations a salt which gave no tests for any of these impurities. The recrystallization of the perchlorate was almost equally effective.

The method of purification finally adopted was, therefore, essentially the following: The fluoride, free from sodium but containing possible traces of calcium, magnesium, and sulphate, was converted into nitrate

${ }^{2}$ Richards, This Journal, 27, 104 (1905). 
or perchlorate as described. These salts, free from fluorine, were recrystallized several times, using centrifugal drainage. The efficiency of this method may be shown by the fact that 70 grams of free acid were so completely removed from 400 grams of lithium perchlorate by three recrystallizations that less than a milligram of free acid remained. Since even in the mother liquors from the first recrystallization no calcium, magnesium, or sulphate could be detected by the ordinary tests, there is no doubt that this recrystallized nitrate or perchlorate was exceedingly pure. All silica must have been removed when the fluoride was dissolved in acid. The final step of this sequence of operations alone remained to be taken, namely, the conversion of the salts into chloride.

In the case of the nitrate the conversion into chloride was not difficult. The solution was poured into a hot, concentrated solution of ammonium carbonate, which had been distilled in a platinum retort, and the precipitated lithium carbonate was washed several times with hot water, using centrifugal drainage. It is essential that hot and concentrated solutions be used; otherwise, the yield of carbonate-at best only 50 per cent.-will be much smaller. The precipitate is coarsely crystalline and easily washed. This process also serves as a means of further purification. The carbonate was dried at $300^{\circ}$, dissolved in slightly less than the theoretical amount of the purest hydrochloric acid which had been twice distilled and condensed and collected in quartz, and subsequently filtered from excess of carbonate. The solution contained a little nitrate, and to convert this into chloride without fear of attacking the platinum, a solution of pure hydrazine hydrate, distilled in platinum, was added, and then excess of hydrochloric acid. After boiling the solution a few minutes, all the nitrate was reduced, and there remained a solution of pure lithium chloride with a little hydrochloric acid and hydrazine chloride, both of which were completely volatilized in subsequent operations. The chloride was recrystallized once or twice, and dried, and it was then ready for analysis. During the purification it was treated only with materials prepared in quartz or platinum.

The conversion of lithium perchlorate into chloride was not so simple. At first the solution was mixed with ammonium chloride and the crystals of ammonium perchlorate were separated by filtration. The solution containing the lithium chloride was evaporated and ignited, but unfortunately, on ignition the small amount of perchlorate remaining caused the evolution of considerable chlorine, which attacked the platinum badly. If quartz was used, it also was attacked, the salt becoming alkaline. Careful ignition in platinum was the method finally adopted, the presence of the metal being decidedly the lesser evil; but it was necessary to regulate the temperature very carefully, otherwise the action became too violent. The chief action on the platinum occurs only towards 
the end of the operation, when a higher temperature was required. It was found advantageous to halt the operation at the point where the mass became pasty, to dissolve the product in water and evaporate to dryness, thus uniformly diluting the perchlorate with an excess of chloride. Sometimes chloride from a previous operation was added. If the final heating was performed in air, the platinum was always slightly attacked, but if this were done in an atmosphere of hydrogen, the slightly alkaline chloride could be fused without any appreciable effect on the vessel. The chloride thus prepared was freed from platinum and crystallized two or three times and was then ready for analysis. This method of preparation must have given a product absolutely free from traces of other halogens. In all the preparation work, exclusion of dust was the greatest difficulty. It was found desirable to shorten all the processes whenever this could be done, so as to expose the material as little as possible. The principal impurity introduced by dust is sodium. It seemed best, therefore, to effect the elimination of the last traces of sodium towards the end of the purification, rather than at the beginning.

Several samples of lithium chloride were prepared by these methods, platinum ressels being used throughout. The material which served for the preparation of sample A consisted of chloride prepared from commercial carbonate and freed from most of its sodium by treatment with alcohol and ether. It was recrystallized six times, precipitated as fluoride and added to another specimen of fluoride which had been precipitated from the mother liquors, twice recrystallized as perchlorate, and finally precipitated yet again as fluoride. Subsequently, it was all converted into perchlorate, twice recrystallized, treated with ammonium chloride to remove most of the perchlorate, and ignited in platinum. The platinum which was dissolved was removed by a solution of hydrazine, and the chloride was crystallized once more from hydrochloric acid solution. Owing to an accident in which most of it was lost, this sample was sufficient for only one analysis. It contained a barely visible trace of sodium.

Sample B was prepared from commercial lithium chloride, also purified by solution in alcohol and ether. The main portion was twice precipitated as fluoride. To this precipitate was added some fluoride precipitated from the last mother liquors of $A$, and the whole was converted into perchlorate, which was recrystallized three times. It was then treated with ammonium chloride, and finally fused in platinum. The platinum which was dissolved was removed as usual by precipitation with hydrazine, and the salt was evaporated to dryness. It also contained a trace of sodium.

Sample D. To the mother liquors from the last three recrystallizations of sample $B$ were added some fairly pure residues of lithium chloride, and the whole was precipitated as fluoride. At this point it was free from 
sodium. After conversion into perchlorate it was recrystallized six times, ignited to chloride in platinum, fused in hydrogen, and twice 1ecrystallized as chloride. This was $D_{3}$. The mother liquors from these two recrystallizations formed $\mathrm{D}_{4}$. The mother liquors from the last three recrystallizations of the perchlorate were once recrystallized; the perchlorate was ignited to chloride and crystallized once. This was $D_{2}$. The mother liquor formed $\mathrm{D}_{1}$. All these preparations contained a trace of sodium, in spite of the fact that previously the material had been free from this element, and no glass vessels had been used. The sodium undoubtedly came from traces of dust.

Sample C. Residues of lithium nitrate and bromide from other work on lithium were treated with ammonium oxalate to remove calcium, and twice precipitated as fluoride. The remainder of sample $B$ was likewise precipitated as fluoride and added to this. All of it was free from sodium. It was converted into nitrate by evaporation in a platinum retort with the purest nitric acid and water, freshly distilled. After expelling all the acid, it was dissolved in water and filtered from a slight residue containing fluoride, and platinum. The solution contained no platinum, and was absolutely free from sodium. The nitrate was recrystallized three times, poured into freshly distilled ammonium carbonate, and the precipitate washed and dried. It was almost completely dissolved in the purest hydrochloric acid, filtered, more acid added, and then a little hydrazine hydrate. Boiling soon destroyed the nitrate present, and the pure chloride was crystallized once. At last, this sample was entirely free from sodium, and therefore was worthy to form the basis of some of our best experiments.

Sample $\mathrm{E}$ was prepared in much the same way as C. Two kilograms of Kahlbaum's best lithium chloride, nearly anhydrous, were available as raw material. Tested carefully, it showed no potassium, calcium or magnesium, and only small traces of iron, but as usual it contained considerable sodium. The salt was dissolved in about 8 liters of pure water, and agitated with pure lithium carbonate, being allowed to stand over night in large Jena flasks. The precipitate contained iron but no calcium. The perfectly clear, colorless solution after filtration was evaporated in a platinum dish, and crystallized with centrifugal draining, and the mother liquor was evaporated further, the second group of similarly drained crystals being added to the others. The whole was recrystallized, and treated again in the same way. In order to eliminate the small amount of sodium which still remained, the lithium was precipitated as fluoride. The coarse-grained, easily washed precipitate was collected upon a Büchner funnel, and was washed two or three times, being each time stirred up with the water. The washed material was whirled in the platinum centrifuge, and then washed six times, taking four milliliters of 
water each time, and draining completely with centrifugal action after each washing. The last wash water contained only a faint trace of chloride and the salt was found to be almost wholly free from sodium when examined in the spectroscope. In this way were obtained 620 grams of dry, very pure lithium fluoride, which served as a basis for yet further operations. These successfully removed the traces of impurity still remaining, as the following description shows.

The coarse-grained powder was now decomposed by heating with a large excess of nitric acid in successive additions. About six times the theoretical quantity of 70 per cent. nitric acid was required in all, diluted with an equal bulk of water, it having been found that the diluted acid was more efficient than the concentrated acid. After the expulsion of the nitric acid, the nitrate was slightly alkaline, and no trace of platinum was found by the potassium iodide test in the filtrate, although the residual precipitate of lithium fluoride contained platinum. In order to be sure that no platinum was present, the clear filtrate was treated with a little hydrazine hydrate, but no change occurred. All the nitrate was finally evaporated with excess of nitric acid until neutralized, and the nitrate was crystallized three times with centrifugal drainage in platinum. The trace of sodium present in the fluoride had now entirely been eliminated. The mother liquors from the last two crystallizations were evaporated and twice recrystallized again, yielding material free from sodium, like the other crystals to which they were added.

The lithium was now precipitated as carbonate-a process which rendered certain the elimination of any traces of fluoride which may have found its way through the successive recrystallizations of the nitrate.

The ammonium carbonate for this purpose was prepared by distilling the substance with water in a platinum still into a platinum dish. The solution was heated to the point of obvious decomposition and the lithium nitrate was then poured into it, the whole being digested for a time at a high temperature. The lithium carbonate was collected in platinum funnels, washed three times with hot water, using centrifugal drainage, and was dried at over $400^{\circ}$ in an electric oven. Only about 250 grams of carbonate were thus obtained, a yield of less than 30 per cent. of the original fluoride.

The lithium carbonate was then dissolved in hydrochloric acid which had been twice distilled in quartz, rejecting the first and last portion each time. The solution was alkaline, because not quite enough acid had been added to correspond to the carbonate. The filtered solution was treated with a small amount of hydrazine hydrate and a considerable excess of hydrochloric acid. After evaporation, the solution which had originally given a strong test for nitrate with diphenylamine was found to be wholly free from this contamination. The purest water was now 
added, enough to make a hot saturated solution, and the substance was crystallized by cooling the platinum vessel in ice. The crystals were drained centrifugally in platinum and another crop was obtained from the mother liquor, and the combined material was redissolved and again recrystallized in the same way. In the least pure of the mother liquors an exceedingly faint trace of sodium was found, but the crystals after centrifugal drainage were entirely free from it as far as the most careful spectroscopic testing could show.

Most of the water was driven off from these crystals over the electric hot plate, and the stirred mass was heated in the electric oven at a high temperature until nearly all the ammonium chloride present had been volatilized. The mass weighed about 170 grams when dry, or about 17 per cent. of the lithium originally present as fluoride. A portion of this substance was broken into pieces in a clean warm agate mortar and used in Analyses $2 \mathrm{I}-22,30$, and $3 \mathrm{I}$.

In spite of the fact that various methods had been used in these purifications, and the fact that some of them contained minute traces of common salt, the five preparations were all essentially identical, except from the point of view of the most critical experimenter. The maximum variation affected only the third decimal place in the atomic weight; the second figure was never in doubt. The two purest samples, which were wholly free from sodium, namely samples $\mathrm{C}$ and $\mathrm{E}$, gave exactly the same result, within the limit of error of the experiments. This fact will become manifest when the detailed figures are presented, on pages 31 and 33 .

\section{The Drying and Weighing of the Lithium Chloride.}

The final preparation of the salt for analysis consisted in expelling the last traces of water by fusion in a platinum boat in a current of dry hydrochloric acid gas and nitrogen, the operation being conducted in the apparatus usually used for this purpose at Harvard. ${ }^{1}$ To avoid absorption of acid by the chloride, this gas was swept out by a current of pure nitrogen, before the salt solidified, and after cooling, the nitrogen was replaced by dry air. The boat was then transferred to the weighing bottle without contact with a trace of moisture, and weighed.

In the preliminary experiments the old form of "bottling apparatus"1 was used for this purpose, but in the final series, the new form shown in Fig. 2 was employed.

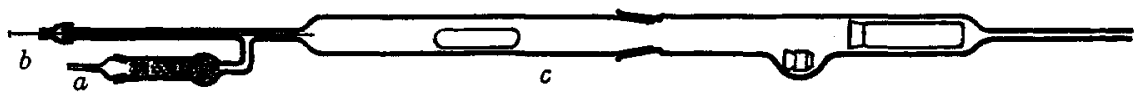

Fig. 2.

In this any possibility that moisture might gain access to the salt is excluded; the calcium chloride tube $(a)$ is not removed and the glass rod used to push the boat into the bottle is passed through a short piece of

${ }^{1}$ Richards and Parker. Proc. Amer. Acad.. 32. 59 (1896). 
rubber tubing at $(b)$. When this apparatus was constructed of glass, evidence was found of the introduction of other alkali-metals into the lithium salt by the action of hydrochloric acid on the red hot tube. Hence in the final experiments, this portion of the apparatus ( $a$ to $c$ ) was made entirely of transparent quartz, and carefully ground into the other. The tube was heated by winding it with "nichrome" ribbon, through which a suitable current was passed, an arrangement that not only gave evenly distributed heat, but also allowed the progress of the fusion to be clearly observed. The apparatus was so constructed that by turning the proper stopcocks, either hydrochloric acid, nitrogen, air, or any desired mixture, could be passed through it. These stopcocks were so carefully polished with rouge that a trace of lubricant composed of a mixture of paraffin and pure rubber made them perfectly gas-tight.

The nitrogen was prepared by passing air charged with ammonia over red-hot copper. Carbon dioxide was removed by means of potassium hydroxide solution, and the excess of ammonia, by passing the gas through a series of towers filled with glass beads moistened with sulphuric acid, the first two containing dilute, and the last three, concentrated acid. The last traces of water were removed by means of resublimed phosphorus pentoxide. Nitrogen prepared in this way usually contains a trace of hydrogen, which in this case was a distinct advantage, since it would counteract the effect on the platinum boat of any trace of oxygen that might be present.

The hydrochloric acid gas was prepared by dropping concentrated sulphuric acid into a solution of pure concentrated hydrochloric acid. It was dried by passing through three towers filled with pearls moistened with freshly boiled concentrated sulphuric acid, for it has been shown ${ }^{1}$ that phosphorus pentoxide is unsuitable for this purpose. No sulphuric acid could be detected in the solution obtained by passing into water a large quantity of the gas thus prepared. ${ }^{2}$

The air, which was furnished by a water pump, was purified by passing successively through towers containing potassium hydroxide, silver nitrate and concentrated sulphuric acid, and was finally dried by phosphorus pentoxide. The entire apparatus was of glass, the different parts being fused together, when possible, or else connected by carefully ground joints.

In detail the procedure was as follows: Dry lithium chloride contained in the platinum boat, was placed in the quartz tube and a mixture of nitrogen and hydrochloric acid passed through it for an hour or two, in order to sweep out all the oxygen. The gases issued under slight pressure,

1 Baxter and Hines, This Journal, 28, 779 (I906).

${ }^{2}$ See also Baxter and Hines, Loc. cit., p. 780 . 
the end of the exit tube dipping just below the surface of mercury, covered with water to absorb the acid. The temperature was very slowly raised so as to expel from the salt as much water as possible before fusion. The chloride was maintained in a state of fusion at a red heat from fifteen to thirty minutes, the longer time apparently causing no further change. During this time the surrounding gas consisted principally of dry hydrochloric acid. Bubbles always formed on the bottom of the boat and were removed only by carefully shaking the tube. The hydrochloric acid was then replaced by pure nitrogen, to remove any excess of acid dissolved in the fused salt, and when the issuing gas was neutral, the chloride was allowed to cool. It was quite transparent and colorless. Because fused sodium chloride was found to be essentially free from dissolved nitrogen, ${ }^{1}$ it is reasonable to assume that the similar lithium salt contained no weighable quantity. After the nitrogen had been replaced by dry air, the boat was bottled and placed in a desiccator. No great difficulty was experienced in obtaining weighings agreeing within $0.02 \mathrm{mg}$., using a similar bottle as counterpoise. Of course the bottle containing the empty boat was always filled with dry air before being weighed. The platinum boat was always weighed separately before and after the fusion, and the loss in weight, if the operation had been carefully conducted, was only a few hundredths of a milligram-sometimes none at all. This correction, if added to the weight of silver chloride, had no appreciable effect on the results.

The boat was placed in a three-liter Erlenmeyer flask of Jena glass, with a carefully ground and polished stopper, and about 0.05 or 0.08 liter of water was added. When the chloride was dissolved, the solution was perfectly clear, and it was usually tested for alkalinity by adding a very dilute solution of phenolphthalein, which had been colored very faintly pink by a trace of sodium hydroxide. It is not sufficient to add a colorless solution of the indicator, for the ever-present trace of carbon dioxide decreases the delicacy of the test. The color never became deeper, and on thorough shaking was discharged by the carbon dioxide. Methyl orange showed a perfectly neutral tint. There can be no doubt, therefore, concerning the neutrality of the salt within the narrow limits thus established. About one liter of freshly distilled water was then added to the flask; the boat was lifted out by means of a heavy platinum wire, bent at one end, and was washed seven times in a beaker, the washings being completely transferred to the flask. This method seemed preferable to dissolving the salt in a beaker and transferring it to the flask, for not more than a few milligrams of lithium chloride could have remained on the boat when it was lifted out, and this small quantity could be transferred without any risk of loss.

1 Richards and Wells, This Journal, 27, 5 I3 $_{3}$ (1905). 
The lithium chloride was then ready for analysis. Both of the familiar methods for finding the amount of chlorine were used, namely, the weighing of the precipitated silver chloride on the one hand, and the determination of the necessary amount of silver on the other. These are discussed below in order.

\section{The Precipitation and Weighing of Silver Chloride.}

The precipitation of the silver chloride and all subsequent operations were carried out under red light in the dark room. To the solution of lithium chloride was added exactly the calculated amount of silver dissolved in a moderate excess of nitric acid, the concentrations of the solutions being about decinormal. The mixture was shaken vigorously for ten minutes and allowed to stand over night. The next day it was again shaken, and to it was added the excess of silver nitrate required for complete precipitation-about 0.05 or $0.06 \mathrm{gram}$ of silver per liter. This method diminishes greatly the danger of occlusion of silver nitrate, as Richards and Staehler have shown. ${ }^{1}$ The solution was shaken from time to time during the next day, and after standing until the supernatant liquid was perfectly clear, it was ready for filtration.

In a few of the preliminary experiments, the Gooch perforated platinum crucible with asbestos mat was used, with all the precautions previously adopted in this laboratory. In all others a Gooch-Munroe crucible ${ }^{2}$ was used. The complete removal of silver chloride from the platinum sponge, when preparing for a new analysis, required treatment with concentrated ammonia for at least twelve hours, followed by a very thorough washing. The crucible was always dried over night at $25^{\circ}$, and a perforated platinum plate was placed upon the sponge to prevent rupture by the contraction of the silver chloride as it dried. This crucible was of the special shape recently described by one of us. ${ }^{3}$

The clear solution was poured through the crucible, and the precipitate was washed four times by decantation with a cold acid solution of silver nitrate, about two-hundredths normal, the precipitate being thoroughly agitated by rotary shaking each time. The filtrate and washings were united and were always practically free from excess of chlorine, so that a constant correction of $0.04 \mathrm{mg}$. of silver chloride per liter was applied to them. The precipitate was then washed ten times with very dilute nitric acid, cooled in ice to reduce the solubility of silver chloride, and finally transferred to the crucible by means of a jet of pure, cold water

${ }_{1}^{1}$ This Journal, 29, 632 (1907). Richards and Staehler, Publ. Carnegie Inst, Washington, 69, I5.

${ }^{2}$ Snelling, Ibid., 3I, 456 (J909).

${ }^{3}$ Richards, ThIS JournaL, 3 I, I 146 .

${ }^{4}$ Richards and Wells, Thrs Journal, 27, 487, 517 (1905). See also Baxter, This JOURNAI, 28, I 322 (1906) 
from a hydrostatic wash-bottle. The entire process was conducted under a clean pane of glass to prevent dust from falling into the crucible. The latter was finally wiped with a clean cloth and heated in an electric oven, gradually increasing the temperature to $250^{\circ}$, where it was maintained at least ten hours. After it had been weighed, the main mass of silver chloride was separated from the platinum disk and fused in a covered quartz crucible contained in a larger one of porcelain. Since the cover was transparent, it was possible to free the fused chloride from the bubbles which invariably adhered to the crucible, without danger of loss from spattering, by carefully rotating the crucible. With one or two exceptions, the fused silver chloride was perfectly colorless and transparent, showing the absence of organic dust and occluded silver nitrate. The loss on fusion was very small, never more than a few hundredths of a milligram per gram. The correction for the entire weight of silver chloride was calculated as usual from that of the portion fused, always over 90 per cent. of the total.

The flask was carefully rinsed out with ammonia, to remove any chloride that might have escaped observation. This with the washings, was tested as follows: To the total washings, exclusive of those with dilute silver nitrate, was added 0.07 gram of silver as nitrate, and after the opalescence had appeared, it was dissolved by pouring in the ammoniacal rinsings. The volume was then made up to one liter. A standard solution was prepared, containing in one liter a known amount of chloride, and the same quantity of ammonia and of silver present in the washings. Twenty-five cc. of each solution were pipetted into a nephelometer ${ }^{1}$ tube, 2 cc. of dilute nitric acid added to each and the contents stirred with a carefully cleaned glass stirrer. The tubes were covered with glass caps having a plane top, and allowed to stand from three to five hours, until constancy was attained. If the tubes were not nearly alike, a new standard was prepared. Several comparisons were always made and found to agree within reasonable limits of error only when extreme care was used in preparing the solutions and in excluding dust. By the use of ice-cold wash water, the total nephelometer correction was reduced from over I $\mathrm{mg}$. to about $0.35 \mathrm{mg}$., of ten less, owing to the decrease in solubility of silver chloride. This matter will subsequently be discussed in detail.

The following table gives the results obtained in the preliminary experiments.

The first two analyses have little value since the technique of the process had not then been mastered and the material was purified only by six recrystallizations as chloride, and was known to contain impurities. No. Io was rejected because it was found that all the silver chloride from the previous analysis had not been dissolved out of the Munroe crucible.

${ }^{1}$ Richards and Wells, Am. Chem. J., 3I, 235 (1904). 
Ratio of lithium Chloride to Silver Chloride.

Preliminary Series.

\begin{tabular}{|c|c|c|c|c|c|}
\hline $\begin{array}{c}\text { No. of } \\
\text { a nalysis. }\end{array}$ & $\begin{array}{l}\text { Prepara- } \\
\text { tion of } \\
\text { LiCl. }\end{array}$ & $\begin{array}{l}\text { Weight of } \\
\text { fused Licl, } \\
\text { vacuum. }\end{array}$ & $\begin{array}{l}\text { Weight of } \\
\text { fused } \mathrm{AgCl} \text {, } \\
\text { vacuum. }\end{array}$ & $\frac{\mathrm{LiCl}}{\mathrm{AgCl}}$ & $\begin{array}{c}\text { Atomic weight } \\
\text { of } \mathrm{Li} \text {. } \\
\mathrm{Ag}=107.88 .\end{array}$ \\
\hline I & 0 & 4.01994 & I 3.59125 & 0.295774 & $6.93^{8}$ \\
\hline 2 & 0 & 6.32840 & $21 \cdot 39635$ & 0.295770 & 6.939 \\
\hline 3 & A & 8.99620 & $30.4 \times 341$ & 0.295797 & 6.942 \\
\hline 4 & B & 4.66824 & $\mathrm{I} 5.78 \mathrm{III}$ & 0.295812 & 6.944 \\
\hline 5 & B & 5.43032 & 18.35734 & $0.2958 \mathrm{I} 2$ & 6.944 \\
\hline 6 & B & 5.10725 & 17.26504 & 0.295815 & 6.944 \\
\hline 8 & $\mathrm{D}_{1}$ & $5 \cdot 74000$ & 19.40375 & 0.295819 & 6.945 \\
\hline 9 & $\mathrm{D}_{2}$ & 5.42038 & 18.32417 & 0.295805 & 6.943 \\
\hline II & $\mathrm{D}_{3}$ & 5.21573 & 17.63280 & 0.295797 & 6.942 \\
\hline 12 & $\mathrm{D}_{4}$ & 6.56925 & 22.20716 & $0.295^{817}$ & 6.945 \\
\hline \multirow[t]{2}{*}{13} & $D_{8}$ & 4.84268 & 16.37121 & 0.295805 & 6.943 \\
\hline & & & & & 6.943 \\
\hline
\end{tabular}

In none of these analyses was the material wholly above reproach, for it all contained traces of sodium. Part of this impurity was present in the original salt, and those specimens $\left(A\right.$ and $D_{3}$ ) which show less sodium in the spectroscope gave a lower value for the atomic weight. Part of the sodium, however, may perhaps have come from the glass tube used to contain the boat in which the lithium chloride was fused. This conclusion was drawn from the fact that material thus fused showed in the spectroscope more sodium than it had possessed before. Nevertheless, these preliminary results are far nearer the truth than the work of any other experimenter.

In order to avoid the vitiating circumstances every conceivable precaution was taken in the final series recorded below. Both the samples of lithium chloride used were proved to be free from any visible trace of sodium discernible upon careful spectroscopic examination. This means, of course, a very high degree of purity. Moreover, the platinum boat during the fusion of the salt was contained in a tube of pure quartz. In this way only can a pure dry sample of lithium chloride be obtained, which will show no trace of sodium in the spectroscope.

As a result of these precautions the values of the atomic weight obtained were appreciably lower than those of the preliminary series, and they agreed distinctly better among themselves. The following table explains itself.

Analyses 19 and 20 were omitted because of accidents which rendered them valueless. Experiments $I_{4}$ to $I_{7}$ inclusive had previously been used for determining the weight of silver needed for the exact precipitation. This part of these experiments is recorded in the table on page 33, under the numbers 26 to 29 . 
Ratio of lithium Chloride to Silver Chi,oride.

Final Series.

\begin{tabular}{|c|c|c|c|c|c|}
\hline $\begin{array}{l}\text { No. of } \\
\text { analysis. }\end{array}$ & $\begin{array}{l}\text { Prepara- } \\
\text { tion of } \\
\text { LiCl. }\end{array}$ & $\begin{array}{l}\text { Weight of } \\
\text { fused Lict, } \\
\text { vacuum. }\end{array}$ & $\begin{array}{l}\text { weight of } \\
\text { fused AgCl, } \\
\text { vacuum. }\end{array}$ & $\frac{\mathrm{LiCl}}{\mathrm{AgCl}}$ & $\begin{array}{c}\text { Atomic weigh } \\
\text { of } \mathbf{L i} \\
\mathbf{A g}=107.880 .\end{array}$ \\
\hline I4 & C & 6.28662 & 2 I. 25442 & 0.295779 & 6.9391 \\
\hline I 5 & $\mathrm{C}$ & 5.82076 & 19.67873 & 0.295790 & 6.9407 \\
\hline 16 & $\mathrm{C}$ & 6.70863 & 22.68030 & 0.295791 & 6.9409 \\
\hline 17 & $\mathrm{C}$ & 6.24717 & 2 I. I 2073 & 0.295784 & 6.9399 \\
\hline 18 & $\mathrm{C}$ & $5 \cdot 5005 x$ & 18.59600 & 0.295790 & 6.9407 \\
\hline $2 I$ & $E$ & 8.34531 & $28.2143^{8}$ & 0.295779 & 6.9391 \\
\hline \multirow[t]{2}{*}{22} & $\mathrm{E}$ & 6.65987 & 22.51564 & 0.295789 & 6.9406 \\
\hline & & $45 \cdot 5^{6877}$ & I 54.06020 & 0.295786 & 6.9401 \\
\hline
\end{tabular}

Comparison of these results is highly interesting, and leaves no doubt concerning the atomic weight of lithium. The average result for the atomic weight of lithium, computed from these final results taken together, is 6.940I, with a "probable error" of 0.0002 .

It is pleasing to note that the average, 6.9403 of experiments 14 to 18 inclusive, made with sample $C$ of lithium chloride is almost identical with the average 6.9399 of experiments $2 \mathrm{I}$ to 22 made with Sample E. The difference between the averages is only 0.0004 , and the average of the first five lies between the values given by the last two experiments. The extreme range is only from 6.939 to $6.94 \mathrm{I}$, whereas the extreme range in the preliminary series was from 6.938 to 6.945 .

The agreement of these two samples made from different raw materials and purified in somewhat different ways seems to leave no doubt concerning the validity of the result except insofar as constant errors in the methods of analysis or constant unavoidable impurities are concerned. The former of these causes of uncertainty is rendered highly improbable by the next series of experiments, to which attention is now directed.

\section{The Ratio of Lithium Chloride to Silver.}

A single series of experiments is never convincing in work of this kind; accordingly the preceding determinations were supplemented by others, which determined the exact amount of silver necessary to precipitate the chlorine in weighed amounts of lithium chloride. No important difference in the final value was expected, because sodium and potassium showed none under similar conditions, but the further work had the advantage of making "assurance doubly sure."

In this series of experiments weights of the purest silver, equivalent to those of the lithium chloride, as calculated from the preceding table, were dissolved in nitric acid in a Jena flask provided with a tower of bulbs to prevent loss by spattering. Almost the exact amount of silver could be weighed out directly, by selecting suitable pieces from a large 
assortment of very fine globules; and the last few hundredth of a milligram were added, if necessary, in the form of a standard solution, prepared from the same silver. The solution of silver nitrate was diluted to about I liter and added to the solution of lithium chloride exactly as in the preceding series. Great care was used to transfer every trace of silver from the flask. After standing for a day or two, with occasional violent shaking, the presence of an excess of silver or of chlorine was then determined with the nephelometer, following the procedure adopted by Richards and Wells. ${ }^{1}$

One very important innovation was introduced. The accuracy with which the end-point can be determined by this method is almost inversely proportional to the concentration of the silver halide remaining in solution, because the percentage accuracy of the nephelometer does not increase with increasing cloudiness. It has long been recognized that the titration of bromides is more exact than that of chlorides, because the solubility of silver bromide is less than one-tenth of that of the chloride. These considerations show that any means of decreasing the solubility of silver chloride will increase the precision of the end-point. The solubility of silver chloride in water changes greatly with the temperature, ${ }^{2}$ being over four times as great at $34^{\circ}$ as it is at I. $5^{\circ}$. It seemed worth while, therefore, to try the effect of cooling the solution in ice. Analysis 3 was made to test this point. At this time the weather was quite warm, the temperature sometimes rising above $30^{\circ}$. The solubility was tested on fourteen successive days, the flask being shaken each day. It was found to vary from $2.2 \mathrm{mg}$. to $3.4 \mathrm{mg}$. per liter; the presence of nitric acid was undoubtedly responsible for a higher average value than Koh1rausch's. Opalescences produced by the precipitation of the chloride in such a solution are so intense that it is difficult to estimate accurately their contents in the nephelometer. The flask was then packed in ice, and the solubility again determined for several days. It became very constant, varying from 0.5 to $0.6 \mathrm{mg}$. per liter. Most of the values obtained were about $0.5 \mathrm{mg}$., a figure which begins to be comparable with that for silver bromide at ordinary temperature-0.10 $\mathrm{mg}$. The opalescences were so faint that the slightest excess of silver or of chlorine was unmistakably evident. Richards and Wells estimated that they could detect a difference of $0.02 \mathrm{mg}$. of silver per liter; this limit we have doubtless reduced by cooling. Assuming the weight of lithium chloride to have been accurate to within $0.02 \mathrm{mg}$., there could not have been an error exceeding $0.05 \mathrm{mg}$. in the weight of silver, since the total volume was only two and one-half liters. The determination by this new method of the weight of silver required is evidently the most accurate part of

${ }^{1}$ THIS Jot RNAL, 27, 502 (1905).

'Kohlrausch, Z. physik. Chem., 64, 148 (1908). 
the analysis, and the cause of the variation in results must be sought elsewhere. There is moreover another advantage in this method. Since the concentration of silver chloride in solution is constant, at the constant low temperature, it is possible to calculate with greater exactness than before the amount of a deficiency of either silver or chloride, in terms of the ratio of lengths of the two nephelometer columns giving equal apparent opalescences. This advantage hastens the work.

Only two preliminary experiments were made, for the technique required by the determination is so simple that it is readily mastered. The results of these two preliminary experiments, made with material not perfectly free from sodium, are given below:

Ratio of Lithium Chloride to Silver.

Preliminary Series.

\begin{tabular}{|c|c|c|c|c|c|c|}
\hline $\begin{array}{l}\text { No. of } \\
\text { experi- } \\
\text { ment. }\end{array}$ & $\begin{array}{l}\text { Continu- } \\
\text { ation of } \\
\text { analysis. }\end{array}$ & $\begin{array}{c}\text { Prepara- } \\
\text { tion of } \\
\text { IiCl. }\end{array}$ & $\begin{array}{l}\text { Wt. fused } \\
\text { Licl, } \\
\text { vacuum. }\end{array}$ & $\begin{array}{l}\text { Wt, of } \Delta g, \\
\text { vacuum. }\end{array}$ & $\frac{\mathrm{IiCl}_{\mathrm{iCg}}}{\mathbf{A g} .}$ & $\begin{array}{l}\text { A tomic wt. } \\
\text { of } \mathrm{Li} \text {. } \\
\mathrm{Ag}=107.88 .\end{array}$ \\
\hline 23 & 3 & A & 8.99620 & 22.89013 & 0.393017 & 6.942 \\
\hline \multirow[t]{2}{*}{24} & 7 & B & 5.25395 & $13 \cdot 36777$ & 0.393030 & 6.943 \\
\hline & & & & Average, & 0.393023 & 6.942 \\
\hline
\end{tabular}

The results agree almost exactly with the preliminary experiments made by the other method with similar material, as may be seen by referring to the first table on page 30 . As has been said, these preliminary experiments are, of course, entirely neglected in this comparison of final results. All of the preparations used in this preliminary work contained traces of sodium, which would, of course, raise the apparent atomic weight of lithium; and accordingly these early values if included in the calculation would introduce a source of error. The experiments were, nevertheless, of great value to the investigators in the course of the work; therefore they seemed worthy of brief notice.

After the various processes had been mastered, the final series, using utmost care to exclude all sources of error, was undertaken. The exper-

Ratio of IITHIUM ChLoRide to Silver.

Final Experiments.

\begin{tabular}{|c|c|c|c|c|c|c|}
\hline $\begin{array}{l}\text { No. of } \\
\text { anal- } \\
\text { ysis. }\end{array}$ & $\begin{array}{l}\text { Continua- } \\
\text { tion of anal- } \\
\text { ysis No. }\end{array}$ & $\begin{array}{l}\text { Prepara- } \\
\text { tion of } \\
\text { LiCl. }\end{array}$ & $\begin{array}{l}\text { Wt. fused } \\
\text { Licl. } \\
\text { vacuum. }\end{array}$ & $\begin{array}{l}\mathrm{Wt} . \mathrm{Ag}, \\
\text { vacuum. }\end{array}$ & $\frac{\mathrm{LiCl}}{\mathrm{Ag}}$ & $\begin{array}{l}\text { A tomic wt. } \\
\text { of } \mathrm{Li} \text {. } \\
\mathbf{A g}=107.88 .\end{array}$ \\
\hline 25 & & & 5.82422 & 14.82035 & 0.392988 & 6.9386 \\
\hline 26 & 14 & $\mathrm{C}$ & 6.28662 & 15.99687 & $0.39299 \mathrm{I}$ & 6.9389 \\
\hline 27 & I 5 & C & $5.8207^{6}$ & 14.81522 & 0.392997 & 6.9396 \\
\hline 28 & I6 & $\mathrm{C}$ & 6.70863 & 17.07038 & 0.392998 & 6.9397 \\
\hline 29 & I7 & C & $6.247 \mathrm{I} 7$ & 15.89620 & 0.392998 & 6.9397 \\
\hline 30 & . & C & $7 \cdot 75349$ & 19.72977 & 0.392984 & 6.9382 \\
\hline \multirow[t]{2}{*}{$3 I$} & . & $\mathrm{E}$ & 7.99108 & $20.334 I_{5}$ & 0.392988 & 6.9386 \\
\hline & & & 46.63197 & II 8.65894 & 0.392992 & 6.9390 \\
\hline
\end{tabular}


iments were consecutive. The method is simple, and accident easily avoided. Four experiments (Nos. 26 to 29 inclusive) were utilized further by weighing the precipitated silver chloride. This portion of the experiment received in each case another number, and the results are given as experiments $I_{4}$ to $I_{7}$ inclusive in the table on page $3 \mathrm{I}$.

These experiments then point to the number 6.9390 as the atomic weight of lithium, a number possessing a so-called "probable error" of less than 0.0002 . It will be observed that the maximum deviation from the mean of all the experiments was less than $0.00^{\circ}$ in the atomic weight of lithium, a quantity which corresponds to only about one part in 65,000 of the lithium chloride. This is about as high a grade of accuracy as has usually been attained in atomic weight work. It means about onetenth of a milligram in weight of the substance taken, although the error is probably not to be traced to the work but rather to inevitable complications in the chemical part of the various processes, especially to occlusion of soluble salts by the silver chloride.

\section{Discussion of the Final Results.}

Thus two final results have been obtained for the atomic weight of lithium, namely, 6.940 by reference to silver chloride, and 6.939 by reference to pure silver. The close agreement of these values is important evidence of their verity, and there seems to be no doubt that the value 6.94 may be taken with great certainty as representing the true atomic weight of this lightest of the metals, supposing that silver is called 107.88. A change of 0.01 in the atomic weight of silver causes a corresponding change of only 0.0006 in that of lithium.

The reasons for the higher atomic weight found by other experimenters are not far to seek. Stas admitted that his lithium chloride was alkaline, a circumstance which would, of course, have raised the apparent atomic weight, and it is probable that further error was introduced by other impurities. Judging from the difficulties encountered in the present work, his methods of purification must have been entirely inadequate. In those cases in the work of others where the approximately correct value of lithium had been found, the fortunate result must be attributed rather to a compensation of errors than to any special excellence.

The fact that the two series of results gave essentially the same atomic weight of lithium shows that they indicate very nearly the same atomic weight of chlorine, referred to silver, as that found in the work of Richards and Wells. The average of the present work shows that one part of pure silver would give $\frac{0.392992}{0.295786}=1.32864$ parts of chloride, while Richards and Wells obtained I.32867. This shows that the silver must have been pure within one part in forty thousand, a fraction about equal to the 
experimental error of the analysis. One pair of experiments, namely the pair numbered 16 and 28 , gave exactly the value found by Richards and Wells.

Another method of comparing these results is to reduce them both to the ratio of lithium chloride to silver. On this basis, if one part of silver chloride corresponds to 0.295786 of lithium chloride, and one part of silver corresponds to 1.32867 parts of silver chloride, ${ }^{1}$ then one part of silver must correspond to 0.393002 part of lithium chloride. This obviously differs by one part in 40,000 from the result 0.392992 found directly. The mean of these two figures, namely 0.392997 , may perhaps be supposed to represent the true value of the ratio to within one part in 80,000 . This value will be chosen for the computation involved in the subsequent work.

\section{Summary.}

In conclusion of the first part of the work, the following brief summary presents the chief features. Lithium salts were in the first place studied with great care in order to discover the most suitable substance for the investigation and the best methods of purifying them. The precipitation of lithium as fluoride and the conversion of this precipitate into nitrate or perchlorate with many recrystallizations of the soluble salts was found to be the surest method of eliminating impurities, especially sodium. The perchlorate was decomposed by heat; the nitrate precipitated by ammonium carbonate in order to convert it into chloride. The purer the material the lower was the observed atomic weight of lithium, and the two purest samples gave precisely the same results within the limit of error of experimentation. In all 46.56877 grams of lithium chloride yielded I54.0602 grams of silver chloride, corresponding to 6.940 as the atomic weight of lithium. Moreover, 46.63197 grams of lithium chloride were found exactly to precipitate I 8.65894 grams of silver, corresponding to the value for the atomic weight 6.939. These essentially identical results may be taken to represent the atomic weight of lithium, if silver is taken with the International Committee to be 107.88. If each of these results is given equal weight and 100.000 parts of silver are assumed to produce 132.867 parts of silver chloride, the corresponding amount of lithium chloride is 39.2997 parts.

\section{PART II.-THE RATIO OF OXYGEN TO LITHIUM CHLORIDE. \\ Preliminary Experiments.}

The reasons which led to the selection of lithium perchlorate as the salt to be used in determining the ratio between oxygen and silver, and the advantages of this substance, have already been mentioned briefly in the

${ }^{1}$ Richards and Wells, Publ. Carnegie Inst. of Washington, 28, 65 (1905). 
introduction. Part I of this paper ${ }^{1}$ has described the determination of the atomic weight of lithium-an essential link in the chain of data.

After lithium perchlorate had been found to be susceptible of fusion without decomposition--thus being rendered anhydrous and suitable for accurate weighing - a method had to be devised for obtaining experimentally the ratio of perchlorate to chloride, and hence the ratio of oxygen to the chloride and to metallic silver.

Naturally, the first attempts were directed toward the quantitative conversion of perchlorate into chloride. Simple ignition first suggested itself, but was quickly abandoned, because this process involves a loss of chloride, unless extraordinary precautions are taken. The oxygen evolved carries with it particles of the salt so extremely minute that they color a flame fifteen or twenty feet distant, if the operation is performed in an open dish. To retain this dust would require such an elaborate apparatus that accurate weighing would be out of the question.

A means of reducing the perchlorate was then sought, which would prevent this evolution of oxygen, but this proved futile, for, as Kreider has shown, ${ }^{2}$ perchloric acid is, in combination, one of the most stable of the oxygen acids, and is hardly affected even by the strongest reducing agents. Hydrogen is entirely without action on the fused salt at $300^{\circ}$, and hydrochloric acid was found to displace only very little of the perchloric. In aqueous solution the situation is not much more promising. Although chlorates in solution are readily reduced, perchlorates under the same conditions are almost entirely unaffected by the most powerful reducing agents, with the exception of ferrous hydroxide and titanous salts, which, in large excess, and upon long boiling, reduce them to chlorides. $^{3}$ This method is, however, quite unsuitable for precise work. All attempts to convert perchlorate into chloride, therefore, hat to be abandoned, and the reverse process was considered.

The vapor from a constant-boiling mixture of perchloric acid and water, both alone and mixed with additional steam, was passed over lithium chloride at different temperatures; but although some hydrochloric acid was expelled, the conversion into perchlorate was only partial. Frothing and creeping occurred, with unavoidable loss of material. The anhydrous acid was not tried, for its use is highly inconvenient or even dangerous; and after an examination of the thermochemical relations involved, there was no reason for supposing that it would be any more effective than its hydrate, which has a higher boiling point. Recourse was had, therefore, to a method similar to that used by Richards and

1 See the preceding pages.

${ }^{2} \mathrm{Am}$.J. Sci, 50, 287 (1895); Z. anorg. Chem., 10, 277.

${ }^{3}$ Sjollema, Z. anorg. Chem., 42, x27 (Ig04). Rothmund, Ibit., 62, I08 (Ig09). 
Forbes ${ }^{1}$ in the synthesis of silver nitrate. Lithium chloride was dissolved in water in a quartz flask, a slight excess of perchloric acid was added, over and above the amount needed to convert the salt wholly into perchlorate, and the solution was evaporated by allowing a current of air to pass through the flask, the temperature being regulated so that no ebullition occurred. The hydrochloric acid was soon expelled, and by gradually raising the temperature the excess of perchloric acid was volatilized, leaving pure, fused lithium perchlorate. The substances in the flask were thus changed gradually from a solution of the chloride into pure fused perchlorate, without the formation of a single bubble of gas or vapor. Hence the process is capable of the greatest accuracy.

Further experiments, planned in order to test details of the operation, showed that pure, neutral perchlorate, prepared by recrystallization, could be heated to $300^{\circ}$ without decomposition. On the other hand, the salt formed in the above process always contained a trace of chloride and chlorate-as shown by its giving off oxides of chlorine when moistened with perchloric acid. This small amount, however, did not increase on further heating-a fact which indicated that it must have been formed during the evaporation of the excess of acid. This train of circumstances suggested that the impurities might be due to a decomposition of the last traces of acid, which are evolved only at a high temperature and in a nearly anhydrous condition. Since the anhydrous acid decomposes readily, while the dihydrate is very stable, it seemed reasonable to suppose that if the last traces of acid were removed at the lowest possible temperature, and in a current of air saturated with moisture (to prevent the dissociation of the dihydrate), this decomposition ought to be prevented, and a salt almost, if not quite, free from chloride obtained. This was found to be true. The purity of the perchlorate obtained depends upon the care with which the excess of acid is expelled before the temperature attains a high point.

\section{The Conversion of Lithium Chloride into Perchlorate.}

The determination of the ratio of lithium chloride to oxygen thus resolved itself into the following processes: Lithium chloride was to be fused and weighed in a boat just as described in Part I; it was then to be dissolved in a slight excess of perchloric acid; the hydrochloric and perchloric acids were to be driven out by steam; and the fused perchlorate was to be dried at $300^{\circ}$ and finally weighed. The gain in weight of the salt is caused by oxygen alone and gives at once the ratio $\mathrm{O}_{4}: \mathrm{LiCl}$. From the known ratio of lithium chloride to silver, the atomic weight of the last-named element is to be calculated.

The general method having been indicated, the complete details of

1 This Journal, 29, 808 (1907). 
the operation and the apparatus used will now be described. The preparation of very pure perchloric acid and lithium chloride has been already discussed in Part I.

The quartz flasks, of Ioo cc. capacity, which served to conduct the quantitative evaporation, were made especially for the work of Heraeus. They are shown in cross section in Figures 3 and 4 , and may be called evaporating flasks.

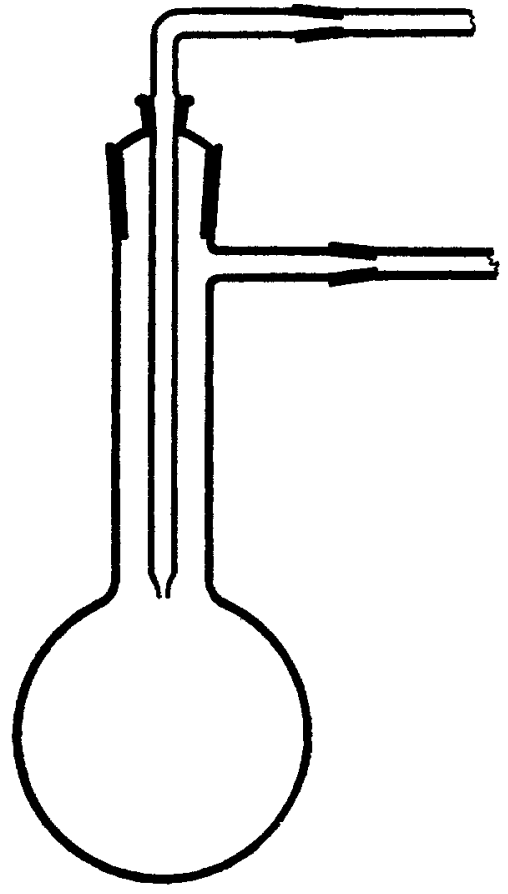

Fig. 3.

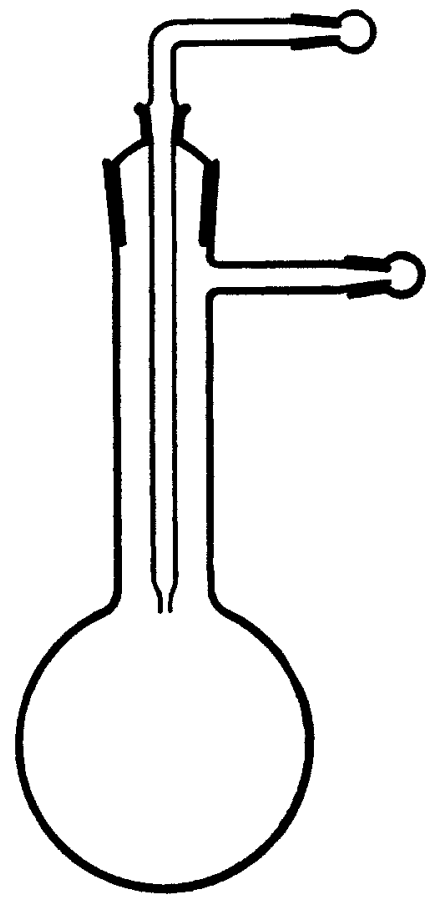

Fig. 4 .

The quartz stopper was provided with a ground joint, into which fitted the bent quartz delivery tube. This tube could be removed and replaced by a small quartz stopper if desired. The ends of the delivery and exit tubes were ground to fit glass tubes (as in Fig. 3), which were attached during the evaporation, and after the operation was finished, were replaced by glass caps, which effectually closed the flask (Fig. 4). A loop of platinum wire fastened around the neck of the flask served to suspend it from the balance while it was being weighed. One of the flasks was used as a counterpoise. They were kept in separate large Hempel desiccators, with sticks of potassium hydroxide, and supported on baskets of platinum wire. Before weighing the empty flask it was always heated to $300^{\circ}$ and dry air was passed through it for a short time, 
in order to remove any adsorbed moisture, and the flask then allowed to cool in a desiccator. As a matter of fact, experiment showed the same weight was obtained if the flask was filled with dry air at the ordinary temperature, but as a precaution it was always heated.

Platinum seemed to accelerate the decomposition of perchlorate by heat, as might have been expected, and the presence of this metal was, therefore, to be avoided. Hence it seemed desirable to fuse the lithium chloride, the accurately weighed factor in the reaction, in a quartz boat, which could then be left in the evaporating flask during the subsequent conversion to perchlorate. The troublesome transfer of its contents would thus be avoided. Careful quantitative experiments showed the lithium chloride could be fused in quartz in a current of hydrochloric acid without attacking the boat in the least, but unless the walls were very strong, it was cracked by the contraction of the fused salt as it cooled. A heavy-walled boat meant a smaller capacity for the fused chloride, and since it was desired to employ the maximum possible amount, the use of quartz was abandoned. A boat of platinum-iridium was especially made to fit in to the flask, and used thereafter for the fusion of the lithium chloride. At one end was a slight projection provided with a hole, through which was passed the platinum wire that served to lower the boat into the flask.

The fusion and weighing of the lithium chloride have already been described in the first part of this paper, and need only be referred to here. The boat containing the fused and carefully weighed chloride was lowered gently into the neck of the evaporating flask to which had just been added a few cubic centimeters of purest water direct from the still. The flask, with the boat suspended in the neck, was placed over an electric heater, covered by a large bell-jar and kept warm over night. The aqueous vapor condensing on the chloride dissolved it, and the solution flowed down into the bulb of the flask. If time were an object, the boat was lowered directly into the water and the contents dissolved in a few minutes, but this method required more water than the other. In either case the final washing was accomplished as follows: To a wash-bottle was attached a glass tube terminating in a capillary, bent sharply at the end at right angles and cut off so close that it was very little thicker than the rest of the capillary and could be passed between the boat and the walls of the flask. By means of this fine horizontal jet of water, the boat could be washed on all sides and within. Since the wash-bottle was of Jena glass, and the water was allowed to remain in it only a few minutes, no appreciable amount of alkali could have been introduced in this way.

The boat was then lifted out and carefully rinsed in a beaker, the washings being tested in the nephelometer for chloride. The amount found 
was never more than a few hundredths of a milligram. This correction, when appreciable, was applied to the weight of lithium chloride.

A slight excess of the purest perchloric acid, which had been condensed and collected entirely in quartz, was now added. The contents of the evaporating flask were thoroughly mixed, and the flask was placed on a support of platinum wire in an electric oven. The oven was made especially for this purpose by winding a three-liter beaker with resistance ribbon, a window being provided through which the flask within could be observed. The stopper and delivery tube were inserted into the flask, and the delivery tube was connected by a ground joint with the source of moist air. The exit tube, passing through a hole in the side of the oven, was connected with a water pump to remove the acid vapors. The oven was provided with a glass cover, suitably perforated to admit the thermometer and the delivery tube.

The air, supplied by a water pump, was purified by passing successively through towers, some containing potassium hydroxide solution with a little manganate, and others containing a fifty per cent. solution of phosphoric acid, to which had been added potassium permanganate. Thus the air was freed from soluble impurities.

When dry air was desired, the current was passed through further tubes containing, respectively, fused potassium hydroxide and resublimed phosphorus pentoxide. When moist air was desired, it was passed through an electrically heated gas washing bottle containing water. By turning the proper stopcocks either moist or dry air could be passed into the evaporating flask. To prevent the condensation of water in the tube, the latter nas wound with resistance wire from the bottle as far as the point connecting it with the quartz flask, and heated by the passage of a suitable current. The temperature of the oven containing the evaporating perchlorate solution was read on a thermometer, the bulb of which was placed in contact with the evaporating flask as close to the fused salt as possible.

The temperature of the oven was kept at $130^{\circ}$ while dry air passed through the flask until about two-thirds of the water had evaporated; then it was raised to $15^{\circ}$ until most of the hydrochloric acid had been expelled. Moist air was at this stage passed through the evaporating flask, and soon perchloric acid was evolved. After several hours at $160^{\circ}$ no more appeared; at $\mathrm{I} 80^{\circ}$ a little more was expelled, and from this point the temperature was slowly raised to $25^{\circ}$. The salt was then neutral but not quite anhydrous. To remove the last traces of water, it was heated in dry air at $280-300^{\circ}$ for at least five hours; further heating was found to cause no diminution in weight. The entire operation usually occupied three days. The perchlorate was allowed to cool in the current of dry air, until the crystals began to appear. It was then 
warmed to the freezing point $\left(26^{\circ}\right)$ and allowed to cool very slowly, so as to avoid too violent solidification. It was quite crystalline and transparent. The glass caps were then placed over the ends of the tubes and the flask was quickly removed to the desiccator, which was placed for a sufficient time beside the balance case in a room kept at nearly constant temperature.

The possibility that solid material which ought to be weighed might be carried away with escaping gas and vapor during the evaporation was duly considered. Experiments already made with silver nitrate ${ }^{1}$ make it unlikely that this was the case. The method of evaporation seems to be really quantitative. Nevertheless, although no mechanical loss is apparent, there is a conceivable possibility that traces of the lithium perchlorate might be carried away in the state of vapor with the escaping perchloric acid or water at the high temperature. Unlikely as this might seem, it is nevertheless worth testing, and the test was easily applied by adding perchloric acid and water after the fused substance had been weighed and then repeating the evaporation, fusion, and weighing. The interpretation is somewhat complicated by the fact that the true weight of perchlorate can never be attained with perfect accuracy until one knows how large an amount of chloride and chlorate have been formed during the fusion. Rarely were these impurities wholly absent. Nevertheless, the following test is fairly satisfactory.

A neutral solution of nearly i4 grams of pure lithium perchlorate, after various preliminary trials in order to find the best succession of temperatures for the evaporation, was treated with a few drops of an excess of perchloric acid, and the acid was evaporated at $165^{\circ}$ for several hours until the mass solidified. The temperature was now raised to $200^{\circ}$ and kept there until the mass which had partially melted had again solidified; again gradually raised to $245^{\circ}$, and kept there for less than two hours; yet once more gradually raised to $280^{\circ}$ where it was kept for five hours, and finally to $300^{\circ}$ for yet three hours more. After making the correction to vacuum, the lithium perchlorate was found to weigh $13.92425 \mathrm{grams}$. It was then heated to $300^{\circ}$ for five hours more and lost 0.00025 gram. This loss was due to loss of oxygen, however, as shown by the fact that on adding dilute perchloric acid a pale yellow solution was obtained. This was again evaporated rapidly at about $2.10^{\circ}$. Gradually the temperature was raised to $240^{\circ}$ and was kept there until the excess of acid had been expelled and was then heated to $300^{\circ}$ for three hours. The weight was now 13.92419, or only 0.00006 less than the first weight. As the heating had been brief, it was continued at about $300^{\circ}$ for four hours more, when the weight was found to be 13.92416 , essentially the same as before. Upon being dissolved in very dilute perchloric acid no

${ }^{1}$ This Journal, 29, 8i 5 (Igo7). 
trace of yellow color was observable, showing that the substance this time had not been greatly decomposed. Nevertheless, in order to test the matter yet again, the fused salt was dissolved in very dilute perchloric acid once more.

The water and perchloric acid were driven off as before, and the residual salt was dried three and a half hours at $300^{\circ}$. This time again the weight had changed only one-thirtieth of a milligram, being I 3.92413 grams; and upon dissolving in dilute acid no trace of yellow color was to be perceived. Thus the total loss in two evaporations amounted to only 0.00012 gram, or an average of about one-twentieth of a milligram for each evaporation. This small loss is negligible, being only I part in 200,000 of the weight of material in question, and even this may be due to nothing more than a slightly greater loss of oxygen before the last weighing than before the first. An accident prevented the determination of the amount of chloride in the final sample. In the carefully finished determinations given in the final table the amount of oxygen lost varied from 0.00009 gram to 0.00078 gram, or on the average about 0.0004 gram. This is considerably more than the divergence between the weights given above, and therefore it is clear that the agreement noted above is as good as could be expected, and shows that no important amount of material is carried away with the stream of vapor and air during evaporation.

\section{The Weighing of Lithium Perchlorate.}

When perfectly neutral, lithium perchlorate is not in the least deliquescent in air of ordinary humidity, but the presence of a very little acid suffices to make it so. After several days' exposure the pure salt is still apparently dry. Nevertheless, great care was taken not to expose the contents of the evaporating flask to the air. The ground stoppers of the flask were always kept closed, and although they were not quite as tight as those on the weighing bottles for the lithium chloride, they were evidently sufficient for the purpose.

In order that the apparent weight of lithium perchlorate might be corrected to a vacuum standard, the density was found by determining the weight of toluene displaced by a known quantity of salt. The toluene had been dried over sodium and then distilled. Its density at $25^{\circ}$ referred to water at $4^{\circ}$ was found to be 0.8617 . Pure lithium perchlorate was fused in a quartz crucible in a current of dry air, broken into coarse pieces and transferred to an Ostwald pycnometer, modified for use with solids. Great care was taken to remove all air bubbles by shaking in a vacuum. The following table records the results:

Weight of $\mathrm{LiClO}_{4}$ in vacuum, grams. 5.2866 5.2866
DENSITY OF I.ITHIUM PERChLORATE.

Weight displaced toluene in yacuum.

I.8757

$\mathbf{r . 8 7 6 0}$ Density of
$\mathrm{I}_{\mathrm{iClO}} \mathrm{iC}_{4} \mathrm{O} / 4$

2.4285

2.4283 
The mean value is 2.428 , and assuming the density of the weights as 8.3 , a correction of 0.00035 I gram was added to every apparent gram of lithium perchlorate.

The weighing of the evaporating flask with its counterpoise proved to be rather troublesome. During the first few minutes on the balance, the flask lost somewhat in weight, frequently as much as $0.20 \mathrm{mg}$.; but eventually the weight became constant. Since both flasks showed this behavior it was not a serious matter, for constancy with respect to one another was all that was. required. After waiting until constancy had been reached, the weight of the flask obtained after replacing it a second time upon the balance was usually within a few hundredths of a milligram of the first one. The initial irregularity may have been due to adsorbed moisture, but was more probably due to slight temperature changes, for with so large a vessel half a degree Centigrade would cause this difference. Hence the temperature of the balance case and the flasks should not change appreciably during the weighing; the more nearly this condition was realized, the more accurate was the result. A large pane of glass was placed between the observer and the balance to prevent the heat of the body from reaching it.

\section{Impurities in the Fused Perchlorate.}

There could have been only four possible impurities in the salt (provided that the lithium chloride and the perchloric acid and water were pure, and that the vessels were not attacked), namely, chloride, chlorate, hydroxide and water. The tests for these will be discussed in order.

After the flask had been weighed, its contents were dissolved in $0 . I$ liter of water, and the chloride present in 25 milliliters was determined in the nephelometer. The amount was always small-usually less than 0.002 per cent.-and the corresponding correction averaged about four-tenths of a milligram in 14.3 grams of perchlorate. The following table contains a statement of the amounts of chloride found in each of the final experiments, and a correction which was, applied to the final weight of lithium perchlorate containing these impurities in order to allow for the oxygen lost. It is evident that for every 0.425 milligram of lithium chloride found, 0.64 gram of oxygen must have been lost, these quantities being in proportion to the combining weights. In the last determination, involving over 22 grams of perchlorate, the correction was so small as to be practically negligible; and in only one or two cases, in which the heating had probably been somewhat too rapid, did the total correction amount to any serious quantity.

Traces of chlorate were always likely to be present with the chloride, and since the quantity was very small, it was most conveniently estimated nephelometrically after reduction with a solution of pure sulphur dioxide. This gave the total chloride, the amount corresponding to the 
chlorate being obtained by subtracting that previously found as chloride. The correction for chlorate was usually entirely negligible; its amount was determined in four cases, analyses $I, 2,7$ and $I I$. Only in the last of these cases where the amount of lithium chloride found was unusually large did the necessary correction equal one-twentieth of a milligram; it was usually less than one-thirtieth. As a rule the more chloride present, the greater was the amount of chlorate, although the proportionality was not exact. Because one-twentieth of a milligram was only one part in 300,000 of the weight of perchlorate in the case of Experiment Ir, and the amount present did not exceed this fraction in any of the other cases, the correction might have been entirely neglected without introducing appreciable error.

\begin{tabular}{|c|c|c|}
\hline $\begin{array}{c}\text { No. ot } \\
\text { experiment. }\end{array}$ & $\begin{array}{l}\text { Weight of } \\
\text { IiCl found. } \\
\text { Milligram. }\end{array}$ & $\begin{array}{c}\text { Correction applied } \\
\text { to weight of } \mathrm{LiclO}_{4} \\
\text { Milligram. }\end{array}$ \\
\hline $7 \ldots \ldots$ & 0.13 & +0.20 \\
\hline $8 \ldots \ldots$ & 0.50 & +0.75 \\
\hline $9 \ldots \ldots$ & 0.16 & +0.24 \\
\hline Iо...... & $0.2 \mathrm{I}$ & +0.32 \\
\hline I I $\ldots \ldots$ & 0.52 & +0.78 \\
\hline $\mathbf{I} 2 \ldots \ldots$ & 0.06 & +0.09 \\
\hline
\end{tabular}

The formation of alkali was never observed, the solution of the perchlorate being always absolutely neutral to indicators. Even when the salt is completely decomposed into chloride, only a little oxide or hydroxide is formed, and in the specimens obtained in this work where only a trace of any kind of decomposition occurred, the amount of alkali must have been infinitesimal indeed.

Although it seemed probable that the salt dried under these conditions was perfectly anhydrous, the inference could only be verified by complete decomposition, as in the corresponding work of Richards and Forbes on silver nitrate. Lack of time has thus far prevented the completion of this final test, but a comparison of our work with the results of these authors indicates that the amount of water, if any, must be extremely small. They found certainly not over $0.00 \mathrm{I}$ per cent. in silver nitrate dried one hour at $210^{\circ}$, while the lithium perchlorate was heated to $300^{\circ}$ for four or five hours in perfectly dry air. The correction for 0.001 per cent. of water in the perchlorate would raise the atomic weight of silver only 0.002 .

Valuable information concerning the probably anhydrous nature of the salt, corroborating the above conclusions based on analogy, was actually obtained in four cases. During the progress of analysis 8 the lithium perchlorate was heated in the first place for four hours at $280^{\circ}$. Three hours more at about the same temperature caused a further loss of only 0.19 milligram, or 0.002 per cent. This loss, being much less than the weight of oxygen lost by the substance, may have been entirely due 
to the incipient decomposition. At any rate, even supposing the loss had been due to water, one might well infer that further heating could hardly expel more than 0 . I of a milligram additional. As will be seen, this inference was verified later. The ninth experiment was treated in dry air for six hours at $25^{\circ}$, a temperature at which the water would undoubtedly be expelled much less rapidly. Two and a half hours more at a temperature $30^{\circ}$ higher caused the expulsion of about 0.2 milligram, and yet three hours more at the same temperature caused a further loss of but little more than 0 . I milligram. Thus only 0.003 pr cent. of the weight of the salt dried at $252^{\circ}$ was lost by prolonged heating at $285^{\circ}$. Even this loss may have been due chiefly to oxygen, of which 0.24 milligram was shown to be lacking by the presence of 0 . I 6 milligram of lithium chloride in the product.

In Experiments II and I2 higher temperatures were used. After having been dried for four and a half hours at $290-300^{\circ}$, the lithium perchlorate in this experiment weighed 17.84843 grams, and after further drying in a current of pure dry air for three hours at $300^{\circ}$, it weighed 17.84842 , a loss of only 0.01 of a milligram. It seemed unlikely that further heating even at this high temperature would cause any further loss of weight. The last experiment, No. I2, was the most conclusive of all. The salt was first dried for five hours at $280^{\circ}$ and was found to weigh $22.5827 \mathrm{I}$ grams. After three hours further heating at $280^{\circ}$, three hours more at $300^{\circ}$, and a few minutes at $310^{\circ}$, the specimen weighed 22.58275 , a slight gain, but one not exceeding a possible error of weighing. Further heating for one hour at $300^{\circ}$ caused an opposite change of only onethirtieth of a milligram in this salt. The weight accepted as the true one was 22.58273 , and one can hardly believe that this substance still retained an appreciable amount of water. These weighings demonstrated not only the probable absence of water, but also the striking stability of the salt.

The four impurities which might possibly have come from the pure materials employed have thus been discussed in detail. It remains to consider the impurities which might have come from the vessels used and from the gases in contact with the fused materials.

The lithium chloride (forming the starting point of the determination) was fused in a platinum boat. In most of the experiments this boat was weighed before and after the fusion, and was usually found to have lost but little. For example, in Experiment 7 the loss was 0.02 milligram, and in Experiment 8, 0.05 milligram and in Experiments 9 and Io, taken together, only o. or milligram. Thus in four experiments involving the fusion of over 18 grams of lithium chloride the total loss of weight was only 0.08 milligram, or 0.0004 per cent. of the weight of the lithium chloride. On the other hand, in the last experiment, No. 12, which was 
otherwise the best of all, the boat lost much more in weight, because in this case a new boat had been employed to accommodate the unusually large quantity of material. The loss was here 0.3 milligram. There was every reason to believe, because in other cases the platinum was not attacked, that this excessive loss, amounting to 0.003 per cent. of the lithium chloride, was due, at least in part, to iron volatilized from the boat during fusion at high temperature in hydrochloric acid. The boat had indeed been previously cleansed by treatment with ammonium chloride at high temperatures as usual, but apparently the cleansing had been insufficient. The mean weight of the boat was taken as the true one. Even the maximum error resulting from this choice amounts to only one part in 60,000 of the weight of lithium chloride, a quantity which seems often to represent the limit of accuracy in experimental work of this kind. On the whole, the evidence is that the boats were not sufficiently attacked to cause any important constant error in the series of results.

Turning now to the quartz flask, the only other receptacle used during the experiments, it was easy to prove that this was not appreciably attacked during the experiments. In three cases in which no regrinding of joints or alteration in tare weight had been necessary, the following successive tares of the evaporating flask were found: Experiment 8, 0.00106; Experiment 9, 0.00103; Experiment 10, 0.00104. This practically proves that the flasks were not appreciably attacked by the acid lithium perchlorate at $300^{\circ}$. It is of course possible that the flasks gained from the fused mass a weight of lithium equal to the weight of silica dissolved, but this is unlikely, especially because the flasks to-day, after all the determinations, still appear perfectly clear and transparent as they did at first.

Neither sodium chloride, silver chloride, nor silver nitrate were found in the previous investigations ${ }^{1}$ to retain on fusion perceptible amounts of oxygen or nitrogen or argon, and, as the air and nitrogen passed over the lithium chloride and lithium perchlorate in the present experiments were both free from other gases, it is reasonable to infer that these salts also did not contain dissolved gas. The absence of hydrochloric acid from the lithitum chloride which had been fused in the gas, and subsequently while fused heated in a current of pure nitrogen, was proved by complete neutrality of the dissolved product.

From these considerations it would appear that both the lithium chloride and lithium perchlorate were obtained in these experiments in a state of purity as great as is practicable in the present state of work of this kind, and therefore that the conclusions based upon the quantitative results are inferior to none.

${ }^{1}$ Richards and Wells, Publ. Carnegie Inst. Washington, 28, 55, 60 (1905), Richards and Forbes, Ibid., 69, 55 (1907). 
There follows a table containing the four preliminary results which were brought to a satisfactory conclusion. The third and sixth of this preliminary series were vitiated by known experimental errors, due to inexperience with the processes involved. No great accuracy is claimed for these results, but they serve to show that the process was one which was capable of exact work, and afforded the valuable training needed to carry out the six consecutive final experiments which followed. The lithium chloride used in these preliminary experiments was not perfectly pure, being of the same quality as that used in the preliminary experiments of the other series. In calculating the last two columns of the table below, values for the ratio $\mathrm{LiCl}: \mathrm{Ag}$ were chosen which corresponded with the particular samples of slightly impure lithium chloride used in each case, namely, 0.39298 for Experiments I and 2, and 0.39304 for Experiments 4 and 5. Thus a large part of the error due to impurities in the lithium chloride was eliminated:

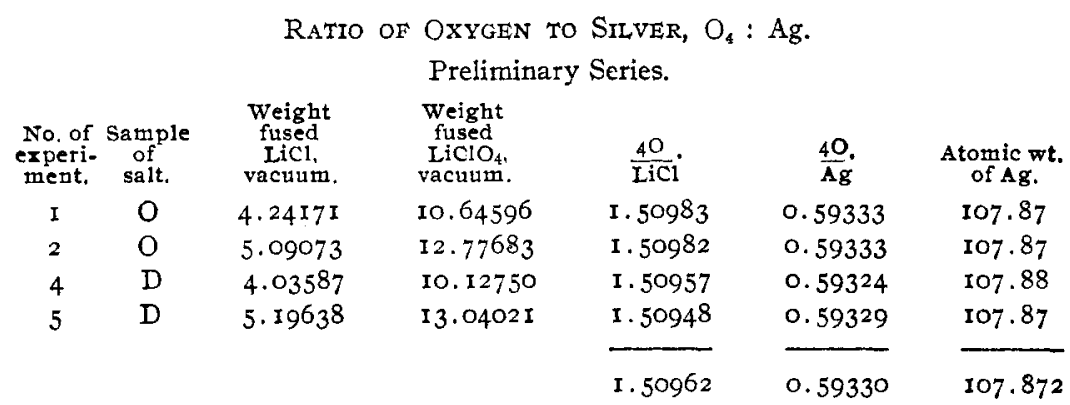

Thus the atomic weight of silver is shown by these preliminary results to be not far from I07.872. It is interesting to note that if Stas's value for the relation of lithium chloride to silver had been used in making the computation of these results, the atomic weight of silver recorded in the last column would have been 107.70 . This value is quite impossible in view of results obtained in other ways, and serves to emphasize the grave error which unquestionably existed in Stas's work on lithium chloride.

These preliminary experiments having furnished an adequate preparation for the execution of an exact series of experiments, six syntheses yielding the following results were made. The lithium chloride used in the determinations was preparation $\mathrm{C}$ already used in previous work involving the comparison with silver. The advantage of using similar material in the two sets of experiments has already been emphasized. Every precaution was taken, and, although we feel that with the experience gained in these experiments, a yet more consistent series might be obtained, especially if somewhat larger quantities of material were 
used; nevertheless, it is evident that this degree of accuracy will not be easy to exceed.

The following table explains itself. The weight of lithium perchlorate given was in each case obtained by adding to the actual weights (corrected to the vacuum standard) the corrections for chloride given in the last column of the table on page 44 , together with the vanishingly small corrections for chlorate mentioned just afterwards. In calculating the values given in the last and next to the last column, the ratio of lithium chloride to silver is assumed to be $0.392997:$ I as previously stated at the conclusion of Part I. No experiment was rejected.

\section{The Ratio of Oxygen to Silver. $\mathrm{O}_{4}$ : Ag.}

Final Series.

\begin{tabular}{|c|c|c|c|c|c|}
\hline $\begin{array}{l}\text { No. of } \\
\text { experiment. }\end{array}$ & $\begin{array}{c}\text { Weight fused } \\
\text { LiCl (vacumm). }\end{array}$ & $\begin{array}{c}\text { Corrected } \\
\text { weight fused } \\
\text { LiC1O, (vacuum) }^{\text {(vecum }}\end{array}$ & $\frac{4 \mathrm{O}}{\mathrm{LiCl}}$ & $\frac{4 \mathrm{O}}{\mathrm{Ag}}$ & $\begin{array}{l}\text { Atomic wt. } \\
\text { of silver. }\end{array}$ \\
\hline 7 & 5.09744 & I 2.79265 & I. 50962 & 0.593276 & 107.876 \\
\hline 8 & 4.20534 & 10.55416 & I. 50970 & 0.593307 & 107.870 \\
\hline 9 & $4 \cdot 54205$ & II. 399 I 2 & I. 50769 & 0.593302 & 107.871 \\
\hline IO & 4.45070 & I I. I 7008 & I. 50974 & 0.593323 & 107.867 \\
\hline II & 7.11167 & 17.84842 & I. 50974 & 0.593323 & 107.867 \\
\hline 12 & 8.99846 & 22.58273 & I. 50962 & 0.593276 & 107.876 \\
\hline Sum. & $=34 \cdot 40566$ & $86.34716 \mathrm{Av}$ & I. 50968 & $0.59330 \mathrm{r}$ & $107.87 \mathrm{I}$ \\
\hline
\end{tabular}

Thus, according to this series of results, silver is $107.87 \mathrm{I}$, if oxygen is taken as 16.000 , and the ratio of lithium chloride to silver is taken as 0.392997 : I. A variation of 4 in the sixth decimal place of this latter ratio would cause a change of only $0.00 \mathrm{I}$ in the atomic weight of silver. There seems to be no doubt that the ratio of lithium chloride to silver is not much more in error than this, and accordingly that the atomic weight of silver given by this series of results is free from error due to the work described in the first part of the present paper. The probable error of the final result, $107.87 \mathrm{I}$, according to the method of least three squares, is 0.003 . The only possibility of serious uncertainty seems to lie in the possible retention of water by the fused lithium perchlorate. This would obviously tend to make the atomic weight of silver appear lower than it is. Accordingly we are disposed to believe that the value 107.87I represents the lower limit. The last analysis, made with the largest amount of material and showing the least decomposition, was probably the most satisfactory determination of all, and it will be noticed that in this case the value is somewhat higher than the average. If silver is $107.87 \mathrm{I}$, lithium becomes 6.939 , essentially the same as if silver were 107.88. Chlorine is somewhat more affected by this difference in silver, sinking from $35 \cdot 457$ to $35 \cdot 454$.

Further determinations carried out as this last determination was, together with other experiments in which the possible presence of water re- 
maining in the final substance should be sought in the manner used by Richards and Forbes in the case of silver nitrate, are needed to settle the third decimal place in the atomic weights of silver and chlorine beyond doubt, but the present investigation seems unquestionably to show that the atomic weights proposed by Stas, both for silver and for lithium, were much too high, and that the recent conclusion of many chemists that the value for silver is not far from 107.88 is entirely justified.

It is worthy of note that this investigation, taken together with that of Richards and Wells, gives values for chlorine, lithium and silver referred to oxygen which are independent of all other work. That these values should agree so closely with other results obtained in such widely different ways is very reassuring as to the degree of precision now within reach.

\section{Summary.}

This investigation consisted in a careful study of three ratios, namely that of lithium chloride to silver chloride, that of lithium chloride to silver, and that of lithium chloride to lithium perchlorate. By means of the last two ratios, $\mathrm{O}_{4}: \mathrm{Ag}$ was calculated, and new values were obtained in this entirely new way for the atomic weights of silver, lithium and chlorine. In the process of this work new methods of purifying lithium salts, better than any preceding, were devised. Lithium chloride was fused in such a way as to show perfect neutrality to the most sensitive indicators, and was weighed in a strictly anhydrous condition. The preparation of perchloric acid also was subjected to rigid scrutiny, and this substance was made in a state of unusual purity. The sharpness of the end-point in the method used for determining the relation between silver and chlorine was much increased by cooling the solution to $0^{\circ}$, in order to diminish the solubility of silver chloride. In other respects the methods of analysis developed in previous Harvard investigations were used in the determination of chlorine. A new precise method was devised for converting lithium chloride into lithium perchlorate, and its sources of error were carefully examined. 45.56877 grams of lithium chloride were found in seven experiments to yield 154.0602 grams of silver chloride on complete precipitation; and in another series of seven experiments partly independent from these 46.63197 grams of lithium chloride were found to need I 8.65894 grams of silver for complete precipitation. In yet another series of six experiments, entirely independent, but using a similar preparation of lithium chloride, 34.40566 grams of this substance were converted into 86.34716 grams of lithium perchlorate. As an outcome of all these experiments, the atomic weight of lithium was found to be 6.939 , and the atomic weight of silver 107.87I, if oxygen is taken as $x 6.000$. 